\title{
Glueball Spectroscopy in Strongly Coupled Lattice Gauge Theories
}

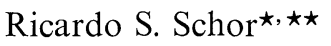 \\ Max-Planck-Institut für Physik und Astrophysik, Werner-Heisenberg-Institut für Physik, \\ D-8000 München, Federal Republic of Germany
}

\begin{abstract}
We study the mass spectrum up to $-7(1-\varepsilon) \log \beta$ of pure threedimensional lattice gauge theories with action $\beta \sum_{P} \chi\left(g_{P}\right)$ for real irreducible $\chi$ and small $\beta$. Besides the lowest excitation $m_{0} \sim-4 \log \beta$, we find two nearly degenerate excited states $m_{1}, m_{2}$ with $m_{i} \sim-6 \log \beta(i=1,2)$ and $\left(m_{1}-m_{2}\right)$ at least $O(\beta)$.
\end{abstract}

\section{Introduction}

The existence of glueballs within QCD has been predicted already some time ago by Fritzsch and Gell-Mann [1]. They are receiving increasing attention, in the context of lattice gauge theories, since the pioneering work of Kogut et al. [2]. The information concerning the mass spectrum in the lattice case has come mainly from Monte Carlo calculations and strong coupling perturbation expansions. See, e.g. $[3,4]$ and references given there. Using appropriate selection rules, excited states have been obtained by locating the lowest excitation within each selection sector, but the methods were not suitable to find states with the same quantum numbers.

In two previous publications $[5,6]$, we started a nonperturbative study of the glueball spectrum in pure gauge lattice models with the Wilson action

$$
S_{\Lambda}=\beta \sum_{P \subset A} \operatorname{Re} \chi\left(g_{P}\right)
$$

making the simplifying assumption that the character $\chi$ is real irreducible and the space-time dimensionality is three (see Sect. 2 for notation). We found isolated one particle states in the full energy-momentum spectrum of the theory, if $\beta$ is small enough. The particle mass $m_{0}(\beta)$ has the asymptotic behaviour $m_{0}(\beta) \sim-4 \log \beta$ as $\beta \rightarrow 0$ and is the only spectrum (besides the vacuum) up to the threshold $-6(1-\varepsilon) \log \beta$.

* Permanent address: Dep. Fisica ICEX-UFMG, 30000 Belo Horizonte, M.G., Brasil

$\star \star$ Work partially supported by CNPq (Brasil) 
In the present paper, we investigate the mass spectrum up to the threshold $-7(1-\varepsilon) \log \beta$ and find precisely two excited states $m_{1}(\beta), m_{2}(\beta)$ with almost degenerate masses : both $m_{1}, m_{2}$ are asymptotic to $-6 \log \beta$ as $\beta \rightarrow 0$, and $\left|m_{1}-m_{2}\right|$ is at least $O(\beta)$. The actual asymptotic behaviour of $m_{1}-m_{2}$ is not determined in this work although the methods developed here are suitable to calculate in principle the power series expansion for the mass difference. These results are derived under the same simplifying assumptions alluded to before but we believe the methods can be adapted to handle more general cases.

We now explain briefly how the excited states are obtained. Precise statements can be found in Sect. 2. The two-dimensional lattice quantum field theory associated to the action (1.1) in three space-time dimensions has an obvious $Z(4)$ symmetry (corresponding to successive rotations $R$ of $\pi / 2$ around an axis parallel to the time direction and through the center of a plaquette). This symmetry induces a selection rule on the zero momentum states which can then be split into a direct sum of four subspaces, each transforming according to the irreducible representations of $Z(4)$. We denote these representations by $E, A_{1}, A_{2}, A_{3}$. They associate to the abstract group $\left\{R^{0}=I, R, R^{2}, R^{3}\right\}$, respectively, $\{1,1,1,1\}$, $\{1,-1,1,-1\},\{1, i,-1,-i\}$, and $\{1,-i,-1, i\}$. The methods developed in $[5,6]$ are suitable to analyze only the lowest non-trivial excitation within each selection sector. In this way, we show that there is no mass spectrum below $-7(1-\varepsilon) \log \beta$ for vectors transforming according to $A_{2}$ or $A_{3}$. For vectors transforming as $A_{1}$, there is exactly one excitation with mass $m_{2}(\beta)$, asymptotic to $-6 \log \beta$ as $\beta \rightarrow 0$. On the subspace corresponding to the identity representation, the method of $[5,6]$ gives only the already known particle $m_{0}(\beta)$. To analyse excited states within this subspace we implemented some ideas developed by Koch in the context of continuum quantum field theories [7]. As it turns out, there is exactly one such state [up to $-7(1-\varepsilon) \log \beta$ ] with mass $m_{1}(\beta)$, which is asymptotic to $-6 \log \beta$ as $\beta \rightarrow 0 ; m_{1}(\beta)$ is obtained as the solution of a "perturbed" equation, whose "unperturbed" solution is $m_{2}(\beta)$. The estimate on the mass difference given above comes from this fact.

The organization of the paper is as follows. In Sect. 2, we give some definitions and present the statements leading to the results above, without proofs. Almost all of them require estimates on decay rates of appropriate Green's functions, which were obtained by using the decoupling procedure of $[5,6]$ extended suitably to handle the region of mass up to $-7(1-\varepsilon) \log \beta$. The required theorems for this part are given in Sect. 3. Finally, in Sect. 4, we give the missing proofs of Sect. 2.

\section{Some Definitions and Main Results}

We consider a $d$ dimensional pure lattice theory with compact group $G$. A gauge field configuration associates to each oriented bound $\ell=\left(x_{1}, x_{2}\right)$ in $\mathbb{Z}^{d}$ a group element $g_{\ell} \in G$, with the convention $g_{\ell^{-1}}=g_{\ell}^{-1}$, where $\ell^{-1}=\left(x_{2}, x_{1}\right)$. If $\varphi$ is a continuous function depending only on a finite number of bond variables $(\varphi$ has "finite support)" we define the expectations

$$
\langle\varphi\rangle\left(\Lambda ;\left\{\beta_{P}\right\}\right)=\frac{1}{Z_{\Lambda}} \int \varphi(g) \exp \left[\sum_{P \subset A} \beta_{P} \operatorname{Re} \chi\left(g_{P}\right)\right] d g_{\Lambda},
$$


where $\Lambda \subset \mathbb{Z}^{d}$ is a finite set containing the support of $\varphi$ and $\chi$ is a character from an irreducible, unitary representation of $G$. The sum in the exponent is over all plaquettes in $\Lambda$, and to each plaquette $P$ we associate a complex number $\beta_{P} ; g_{P}$ is the oriented product of group elements along the boundary of $P$ and $d g_{A}$ is a product of Haar measures, one for each bond in $\Lambda . Z_{\Lambda}$ is a normalization factor, such that $\langle 1\rangle\left(\Lambda ;\left\{\beta_{P}\right\}\right)=1$. From the cluster expansion of Osterwalder and Seiler [8], there are constants $\beta_{0}$ (independent of $\Lambda, \varphi$ ) and $C_{\varphi}$ (independent of $\Lambda$ ) such that (2.1) is analytic and uniformly bounded by $C_{\varphi}$ on $\left|\beta_{P}\right|<\beta_{0}$. Also, truncated correlations have exponential decay rates: if the supports of $\varphi, \psi$ are separated by a distance $d$, then on $\left|\beta_{P}\right|<\beta_{0}$,

$$
\left|\langle\varphi \psi\rangle\left(\Lambda ;\left\{\beta_{P}\right\}\right)-\langle\varphi\rangle\left(\Lambda ;\left\{\beta_{P}\right\}\right)\langle\psi\rangle\left(\Lambda ;\left\{\beta_{P}\right\}\right)\right| \leqq C_{\varphi \psi}^{\prime} e^{-M d}
$$

for suitable constants $M$ (independent of $\Lambda, \varphi, \psi)$ and $C_{\varphi \psi}^{\prime}$ (independent of $\Lambda$ ). In addition, setting all $\beta_{P}=\beta, 0<\beta<\beta_{0}$ the expectations $\langle\varphi\rangle(\Lambda ; \beta)$ converge uniformly to $\langle\varphi\rangle(\beta)$ as $\Lambda \rightarrow \mathbb{Z}^{d}$ and define a probability measure $d \mu$ on the Baire subsets $\Sigma$ of $\mathscr{X}=\prod_{\ell \subset \mathbb{Z}^{d}} \mathrm{G}_{\ell}$ (infinite product of $G$ with itself, one factor for each bond in $\mathbb{Z}^{d}$ ). The "interacting measure" $d \mu$ is translation and time reflection invariant, so that these operations are implemented on the "path space" $\mathscr{E}=L^{2}(\mathscr{X}, \Sigma, d \mu)$ by unitary operators, denoted $U(x), x \in \mathbb{Z}^{d}$ and $\theta$, respectively. The physical Hilbert space is the time zero "slice" of $\mathscr{E}$, i.e. $\mathscr{H}=L^{2}\left(\mathscr{X}, \Sigma_{0}, d \mu\right)$, where $\Sigma_{0}$ is generated by continuous $\varphi$ of finite support in the time zero hyperplane of $\mathbb{Z}^{d}$ and a FeynmanKac formula holds for gauge invariant functions. Thus, for any $\varphi, \psi \in \mathscr{H}$ gauge invariant writing $x=\left(x_{0}, \mathbf{x}\right)$ with $x_{0} \in \mathbb{Z}, \mathbf{x} \in \mathbb{Z}^{d-1}$,

$$
\left(\varphi, U\left(x_{0}, \mathbf{x}\right) \psi\right)_{\mathscr{E}}=\left(\varphi, U\left(-x_{0}, \mathbf{x}\right) \psi\right)_{\mathscr{E}}=\left(\varphi, e^{-H\left|x_{0}\right|} e^{i \mathbf{P} \cdot \mathbf{x}} \psi\right)_{\mathscr{H}},
$$

where $H$ and $\mathbf{P}$ are the energy and momentum operators, respectively. See [6] for more details on this formalism.

In the sequel, we only deal with gauge invariant functions of finite support in the time zero hyperplane. We define

$$
\begin{gathered}
G_{\varphi \psi}(x)=(\varphi, U(x) \psi)_{\mathscr{E}}-(\varphi, 1)_{\mathscr{E}}(1, \psi)_{\mathscr{E}}, \\
\hat{G}_{\varphi \psi}\left(x_{0}\right)=\sum_{\mathbf{x}} G_{\varphi \psi}\left(x_{0}, \mathbf{x}\right)
\end{gathered}
$$

and

$$
\tilde{G}_{\varphi \psi}\left(p_{0}\right)=\sum_{x_{0}} \hat{G}_{\varphi \psi}\left(x_{0}\right) e^{i p_{0} x_{0}}
$$

so that $\tilde{G}_{\varphi \psi}\left(p_{0}\right)$ is the usual Fourier transform of $G_{\varphi \psi}(x)$ at zero momentum. From (2.2), there exists a constant $C_{\varphi \psi}$ such that for $0<\beta<\beta_{0}$,

$$
\sum_{x}\left|G_{\varphi \psi}(x)\right| \leqq C_{\varphi \psi}
$$

Also, from (2.3), $\tilde{G}_{\varphi \psi}\left(p_{0}\right)$ has the integral representation

$$
\tilde{G}_{\varphi \psi}\left(p_{0}\right)=(2 \pi)^{3} \int_{(0, \infty)} \int_{(-\pi, \pi]^{d-1}} \frac{\sinh \lambda_{0}}{\cosh \lambda_{0}-\cos p_{0}} \delta(\lambda) d\left(\varphi, E\left(\lambda_{0}, \lambda\right) \varphi\right)_{\mathscr{H}},
$$


where $d E\left(\lambda_{0}, \lambda\right)$ is the joint energy-momentum spectral measure. Thus, the singularities of (2.8) are located at the spectrum of the energy operator at zero momentum (plotted in the imaginary axis), the poles corresponding to particles. It is the goal of this work to find the possible singularities of $\tilde{G}_{\varphi \psi}\left(p_{0}\right)$ up to the threshold $\left|\operatorname{Im} p_{0}\right| \leqq-7(1-\varepsilon) \log \beta$, for arbitrary $\varphi$. The analysis can be carried out due to the existence of selection rules operating on the zero momentum states. We assume the space-time dimensionality $d=3$ from now on.

From the invariance of the interacting measure under rotations of $\pi / 2$ around an axis parallel to the time direction and through the center of a plaquette, it follows that

$$
G_{\varphi \psi}\left(x_{0}, \mathbf{x}\right)=G_{R_{\mathbf{a}} \varphi, R_{\mathbf{a}} \psi}\left(x_{0}, R \mathbf{x}\right),
$$

where $R_{\mathbf{a}}$ denotes rotation along an axis through $\mathbf{a}$ and $R \mathbf{x}$ is $\mathbf{x}$ rotated by $\pi / 2$ around the origin. Thus, $\hat{G}_{\varphi \psi}\left(x_{0}\right)=\hat{G}_{R_{\mathbf{a}} \psi, R_{\mathbf{a}} \psi}\left(x_{0}\right)$. Actually,

$$
\hat{G}_{\varphi \psi}\left(x_{0}\right)=\hat{G}_{R_{\mathbf{a}} \varphi, R_{\mathbf{b}} \psi}\left(x_{0}\right)
$$

for arbitrary a, b. This is because $R_{\mathbf{b}}=R_{\mathbf{a}} U(\mathbf{z})$ provided $\mathbf{z}=\left(a_{1}-a_{2}+b_{2}-b_{1}\right.$, $a_{1}+a_{2}-b_{1}-b_{2}$ ) and so,

$$
\hat{G}_{R_{\mathbf{a}} \varphi, R_{\mathbf{b}} \psi}\left(x_{0}\right)=\hat{G}_{R_{\mathbf{a}} \varphi, R_{\mathbf{a}} U(\mathbf{z}) \psi}\left(x_{0}\right)=\hat{G}_{\varphi, U(\mathbf{z}) \psi}\left(x_{0}\right)=\hat{G}_{\varphi \psi}\left(x_{0}\right) .
$$

Now, define

$$
\begin{array}{cl}
P_{\mathbf{a}}^{(1)}=(1 / 4)\left(1+R_{\mathbf{a}}+R_{\mathbf{a}}^{2}+R_{\mathbf{a}}^{3}\right), & P_{\mathbf{a}}^{(2)}=(1 / 4)\left(1-R_{\mathbf{a}}+R_{\mathbf{a}}^{2}-R_{\mathbf{a}}^{3}\right), \\
P_{\mathbf{a}}^{(3)}=(1 / 4)\left(1+i R_{\mathbf{a}}-R_{\mathbf{a}}^{2}-i R_{\mathbf{a}}^{3}\right), & P_{\mathbf{a}}^{(4)}=(1 / 4)\left(1-i R_{\mathbf{a}}-R_{\mathbf{a}}^{2}+i R_{\mathbf{a}}^{3}\right) .
\end{array}
$$

Clearly, $P_{\mathbf{a}}^{(i)} P_{\mathbf{a}}^{(j)}=\delta_{i j} P_{\mathbf{a}}^{(i)}$ and $\sum_{i} P_{\mathbf{a}}^{(i)}=1$. Moreover, $\hat{G}_{P_{\mathbf{a}}^{(2)} \varphi, \psi}\left(x_{0}\right)=\hat{G}_{\varphi, P_{\mathbf{b}}^{(i)}}\left(x_{0}\right)$, so that $\hat{G}_{\varphi \psi}\left(x_{0}\right)=0$ if $\varphi=P_{\mathbf{a}}^{(i)} \varphi, \psi=P_{\mathbf{b}}^{(j)} \psi$ with $i \neq j$. This is the selection rule referred to above. It reduces our problem to locating singularities of functions of the form $\tilde{G}_{\varphi_{1} \varphi_{i}}\left(p_{0}\right), 1 \leqq i \leqq 4$ with $\varphi_{i}=P_{\mathbf{a}_{i}}^{(i)} \varphi_{i}$ for some $\mathbf{a}_{i}$. As it turns out, the cases $i=3,4$ are the easiest to analyse [up to $-7(1-\varepsilon) \log \beta]$. Notice that since $\tilde{G}_{\varphi \varphi}\left(p_{0}+2 \pi\right)$ $=\hat{G}_{\varphi \varphi}\left(p_{0}\right)=\tilde{G}_{\varphi \varphi}\left(-p_{0}\right)$, it is sufficient to restrict $p_{0}$ to $\left|\operatorname{Re} p_{0}\right| \leqq \pi, \operatorname{Im} p_{0} \geqq 0$, and in this region the singularities can lie only in the imaginary axis, see (2.8). In the following, we assume $\varepsilon \leqq 1 / 10, \varrho_{0} \leqq 1 / 2$ and the character $\chi$ in (2.1) real irreducible. The reality assumption simplifies the analysis of the problem under investigation. See the beginning of Sect. 3, where we point out these simplifications.

Theorem 2.1. There exists $\beta_{1} \leqq \beta_{0}$ such that for $0<\beta<\beta_{1}, \tilde{G}_{\varphi \varphi}\left(p_{0}\right)$ is analytic on $\left|\operatorname{Re} p_{0}\right| \leqq \pi, 0 \leqq \operatorname{Im} p_{0} \leqq-7(1-\varepsilon) \log \beta$ if $\varphi=P_{\mathbf{a}}^{(i)} \varphi$ for any $\mathbf{a}$ and $i=3,4$.

To analyse $\tilde{G}_{\varphi_{i} \varphi_{1}}\left(p_{0}\right)$ with $i=1,2$, we introduce the function $\chi_{h}(g)=\chi\left(g_{w}\right)$, where $g_{W}$ is the oriented product of the six group elements along the boundary of the elementary horizontal "window" located at the origin of the time zero plane.

Let $\chi_{1}=P_{\mathbf{0}}^{(1)} \chi_{h}, \chi_{2}=P_{\mathbf{0}}^{(2)} \chi_{h}$. As will be apparent from the next few theorems, the study of the analytic structure up to $\left|\operatorname{Im} p_{0}\right| \leqq-7(1-\varepsilon) \log \beta$ of $\tilde{G}_{\varphi_{2} \varphi_{2}}\left(p_{0}\right)$ is completely analogous to the one developed in $[5,6]$ for $\tilde{G}_{\varphi_{1} \varphi_{1}}\left(p_{0}\right)$ up to $\left|\operatorname{Im} p_{0}\right|$ $\leqq-6(1-\varepsilon) \log \beta$, the function $\chi_{2}$ replacing the elementary plaquette $\chi$ of the latter case. Thus, we have 
Theorem 2.2. There exist positive constants $k_{1}, k_{2}, d_{1}, d_{2}$ and $\beta_{2} \leqq \beta_{1}$ such that if $0<\beta<\beta_{2}$,

$$
k_{1}\left(d_{1} \beta\right)^{6\left|x_{0}\right|} \leqq \hat{G}_{\chi_{2} \chi_{2}}\left(x_{0}\right) \leqq k_{2}\left(d_{2} \beta\right)^{6\left|x_{0}\right|} .
$$

We define $m_{2}(\beta)=-\lim _{\left|x_{0}\right| \rightarrow \infty}\left(1 /\left|x_{0}\right|\right) \log \hat{G}_{\chi_{2} \chi_{2}}\left(x_{0}\right)$, so that $\lim _{\beta \rightarrow 0} m_{2}(\beta) /-6 \log \beta=1$ and $\beta_{2}$ can be chosen such that

$$
-\frac{11}{2} \log \beta<m_{2}(\beta)<-\frac{13}{2} \log \beta \text {. }
$$

Let $\hat{\Gamma}_{\chi_{2} \chi_{2}}\left(x_{0}\right)$ be the convolution inverse of $-\hat{G}_{\chi_{2} \chi_{2}}\left(x_{0}\right)$. The existence of $\hat{\Gamma}_{\chi_{2} \chi_{2}}$ is part of the following

Theorem 2.3. There exists $\beta_{3} \leqq \beta_{2}$ such that for $0<\beta<\beta_{3}, \hat{\Gamma}_{\chi_{2} \chi_{2}}\left(p_{0}\right)$ is analytic on $\left|\operatorname{Re} p_{0}\right| \leqq \pi, 0 \leqq \operatorname{Im} p_{0} \leqq-7\left(1-\frac{\varepsilon}{2}\right) \log \beta$.

From this last result, $\tilde{G}_{\chi_{2} \chi_{2}}\left(p_{0}\right)$ is meromorphic on $\left|\operatorname{Re} p_{0}\right| \leqq \pi, 0 \leqq \operatorname{Im} p_{0}$ $\leqq-7(1-\varepsilon) \log \beta$. Using the integral representation (2.8) and (2.11) it is easy to see that $\tilde{G}_{\chi_{2} \chi_{2}}\left(p_{0}\right)$ has precisely one simple pole at $p_{0}=i m_{2}$ (see $[5,9]$ for more details). Thus, $\tilde{G}_{\chi_{2} \chi_{2}}\left(p_{0}\right)$ has the form

$\tilde{G}_{\chi_{2} \chi_{2}}\left(p_{0}\right)=v_{2}\left(m_{2}\right) \frac{\sinh m_{2}}{\cosh m_{2}-\cos p_{0}}+\int_{-7\left(1-\frac{\varepsilon}{2}\right) \log \beta}^{\infty} \frac{\sinh \lambda_{0}}{\cosh \lambda_{0}-\cos p_{0}} d v_{2}\left(\lambda_{0}\right)$

with $v_{2}\left(m_{2}\right)>0$ and $d v_{2}\left(\lambda_{0}\right)=(2 \pi)^{3} \int \delta(\lambda) d\left(\chi_{2}, E\left(\lambda_{0}, \lambda\right) \chi_{2}\right)_{\mathscr{H}}$. We write

$$
\begin{gathered}
\tilde{H}_{1}\left(p_{0}\right)=v_{2}\left(m_{2}\right) \frac{\sinh m_{2}}{\cosh m_{2}-\cos p_{0}}, \\
\tilde{H}_{2}\left(p_{0}\right)=\int_{-7\left(1-\frac{\varepsilon}{2}\right) \log \beta}^{\infty} \frac{\sinh \lambda_{0}}{\cosh \lambda_{0}-\cos p_{0}} d v_{2}\left(\lambda_{0}\right) .
\end{gathered}
$$

Theorem 2.4. There are constants $k_{3}, k_{4}$ and $\beta_{4} \leqq \beta_{3}$ such that for $0<\beta<\beta_{4}$

(a) $0<v_{2}\left(m_{2}\right)<k_{3}$, there.

(b) $\tilde{H}_{2}\left(p_{0}\right)$ is analytic on $\left|\operatorname{Re} p_{0}\right| \leqq \pi, 0 \leqq \operatorname{Im} p_{0} \leqq-7(1-\varepsilon) \log \beta$ and $\left|\tilde{H}_{2}\left(p_{0}\right)\right| \leqq k_{4}$

To show that $p_{0}=i m_{2}$ is the only possible singularity on $\left|\operatorname{Re} p_{0}\right| \leqq \pi, 0 \leqq \operatorname{Im} p_{0}$ $\leqq-7(1-\varepsilon) \log \beta$ of $\tilde{G}_{\varphi \varphi}\left(p_{0}\right)$ for arbitrary $\varphi=P_{\mathbf{a}}^{(2)} \varphi$, we proceed as in [6] defining

$$
\tilde{F}_{\varphi \varphi}^{(2)}\left(p_{0}\right)=\tilde{G}_{\varphi \varphi}\left(p_{0}\right)+\tilde{G}_{\varphi \chi_{2}}\left(p_{0}\right) \tilde{\Gamma}_{\chi_{2} \chi_{2}}\left(p_{0}\right) \tilde{G}_{\chi_{2} \varphi}\left(p_{0}\right) .
$$

By using a spectral representation analogous to (2.8) one shows that $\tilde{G}_{\varphi \chi_{2}}\left(p_{0}\right)$ and $\tilde{G}_{\chi_{2} \varphi}\left(p_{0}\right)$ are analytic in the region above, with the possible exception of a simple pole at $p_{0}=i m_{2}$. Thus, the desired result for $\tilde{G}_{\varphi \varphi}\left(p_{0}\right)$ follows from the next theorem.

Theorem 2.5. Assume $0<\beta<\beta_{3}$. Then, $\tilde{F}_{\varphi \varphi}^{(2)}\left(p_{0}\right)$ is analytic on $\left|\operatorname{Re} p_{0}\right| \leqq \pi, 0 \leqq \operatorname{Im} p_{0}$ $\leqq-7(1-\varepsilon) \log \beta$.

We next study functions of the form $\tilde{G}_{\varphi \varphi}\left(p_{0}\right), \varphi=P_{\mathbf{a}}^{(1)} \varphi$. An analysis up to $\left|\operatorname{Im} p_{0}\right| \leqq-6(1-\varepsilon) \log \beta$ has already been done in $[5,6]$. Thus, in the theorem 
below we just summarize the results, without giving the corresponding proofs in Sect. 4. These could also be obtained by adapting the proofs of Theorems 2.2, 2.3, and 2.5 .

We denote simply by $\chi$ the function corresponding to the elementary plaquette located at the origin of the time zero plane, and by $\tilde{\Gamma}_{\chi \chi}\left(x_{0}\right)$ the convolution inverse of $-\tilde{G}_{\chi \chi}\left(x_{0}\right)$.

Theorem 2.6. There exists $\beta_{5} \leqq \beta_{4}$ such that for $0<\beta<\beta_{5}, \tilde{G}_{\varphi \varphi}\left(p_{0}\right)$ is analytic on $\left|\operatorname{Re} p_{0}\right| \leqq \pi,\left|\operatorname{Im} p_{0}\right| \leqq-6(1-\varepsilon) \log \beta$ except possibly for a simple pole at $p_{0}=i m_{0}(\beta)$. Here $\varphi=P_{\mathbf{a}}^{(1)} \varphi, \quad m_{0}(\beta)=-\lim _{\left|x_{0}\right| \rightarrow \infty}\left(1 /\left|x_{0}\right|\right) \log \tilde{G}_{\chi x}\left(x_{0}\right)<-5 \log \beta$, and in fact $\lim _{\beta \rightarrow 0} m_{0}(\beta) /(-4 \log \beta)=1$. In addition, $\tilde{\Gamma}_{\chi \chi}\left(p_{0}\right)$ is analytic in the region above.

To investigate the analytic structure on $-6(1-\varepsilon) \log \beta \leqq \operatorname{Im} p_{0} \leqq-7(1-\varepsilon) \log \beta$ for general $\tilde{G}_{\varphi_{1} \varphi_{1}}\left(p_{0}\right)$ we consider first $\tilde{G}_{\chi \chi}\left(p_{0}\right)$. We will show, to begin with, that $\tilde{\Gamma}_{\chi \chi}\left(p_{0}\right)$ is analytic on $\left|\operatorname{Re} p_{0}\right| \leqq \pi, 0 \leqq \operatorname{Im} p_{0} \leqq-7(1-\varepsilon) \log \beta$ except for a simple pole at $p_{0}=i \varrho,\left|m_{2}-\varrho\right|=O(\beta)$. With additional estimates, we then show that $\tilde{\Gamma}_{\chi \chi}\left(p_{0}\right)$ has also a simple zero nearby, at $p_{0}=i m_{1}$ with $\left|m_{1}-m_{2}\right|=O(\beta)$. Thus, $\tilde{G}_{\chi \chi}\left(p_{0}\right)$ has two simple poles, at $p_{0}=i m_{0}$ and $p_{0}=i m_{1}$. We next show that this is also the case for $\tilde{G}_{\chi_{1} \chi_{1}}\left(p_{0}\right)\left(\chi_{1}\right.$ as defined before $)$ and finally extend the result for general $\tilde{G}_{\varphi_{1} \varphi_{1}}\left(p_{0}\right)$. Let

$$
\tilde{F}_{\chi_{1} \chi_{1}}\left(p_{0}\right)=\tilde{G}_{\chi_{1} \chi_{1}}\left(p_{0}\right)+\tilde{G}_{\chi_{1} \chi}\left(p_{0}\right) \tilde{\Gamma}_{\chi \chi}\left(p_{0}\right) \tilde{G}_{\chi \chi_{1}}\left(p_{0}\right)
$$

Since $\chi$ is expected to couple to all excited states with the same quantum numbers, a naive analysis using the spectral theorem suggests that the subtraction from $\tilde{G}_{\chi_{1} \chi_{1}}$ performed in (2.15) should get rid of all physical poles (i.e. poles corresponding to particles) in the function $\tilde{F}_{\chi_{1} \chi_{1}}$. The possible singularities of $\tilde{F}_{\chi_{1} \chi_{1}}$ therefore should be related to those of $\tilde{\Gamma}_{\chi \chi}$. The next few theorems shows that this is indeed the case.

Let $\hat{\Phi}_{\chi_{1} \chi_{1}}\left(x_{0}\right)$ be the convolution inverse of $-\hat{F}_{\chi_{1} \chi_{1}}\left(x_{0}\right)$. Its existence is proven as part of the

Theorem 2.7. There exists $\beta_{6} \leqq \beta_{5}$ such that for $0<\beta<\beta_{6}, \tilde{\Phi}_{\chi_{1} \chi_{1}}\left(p_{0}\right)$ is analytic on $\left|\operatorname{Re} p_{0}\right| \leqq \pi, 0 \leqq \operatorname{Im} p_{0} \leqq-7(1-\varepsilon) \log \beta$.

Thus, $\tilde{F}_{\chi_{1} \chi_{1}}\left(p_{0}\right)$ is meromorphic on the region above. From the work in [6], the poles can only occur for $\operatorname{Im} p_{0}>-6(1-\varepsilon) \log \beta$. To find them, we introduce the function

$$
\tilde{K}\left(p_{0}\right)=\tilde{\Gamma}_{\chi_{2} \chi_{2}}\left(p_{0}\right)-\tilde{\Phi}_{\chi_{1} \chi_{1}}\left(p_{0}\right)
$$

As will be seen, $\tilde{K}$ play a role analogous to the Bethe-Salpeter kernel in [7]. Define also

$$
\tilde{T}_{i}\left(p_{0}\right)=\tilde{K}\left(p_{0}\right) \tilde{H}_{i}\left(p_{0}\right), \quad i=1,2
$$

[see (2.13) and (2.14)].

Theorem 2.8. There are constants $k_{5}$ and $\beta_{7} \leqq \beta_{6}$ such that for $0<\beta<\beta_{7}$,

(a) $\tilde{K}\left(p_{0}\right)$ is analytic on $\left|\operatorname{Re} p_{0}\right| \leqq \pi, 0 \leqq \operatorname{Im} p_{0} \leqq-7(1-\varepsilon) \log \beta$ and $\left|\tilde{K}\left(p_{0}\right)\right|$ $\leqq k_{5} \beta^{\left(\frac{7}{2} \varepsilon\right)}$ there.

(b) $\left|\tilde{T}_{2}\left(p_{0}\right)\right| \leqq \frac{1}{2}$ on the region of (a). 
From the definition (2.16),

$$
\tilde{F}_{\chi_{1} \chi_{1}}\left(p_{0}\right)=\frac{\tilde{G}_{\chi_{2} \chi_{2}}\left(p_{0}\right)}{1+\tilde{K}\left(p_{0}\right) \tilde{G}_{\chi_{2} \chi_{2}}\left(p_{0}\right)}=\frac{\tilde{H}_{1}\left(p_{0}\right)+\tilde{H}_{2}\left(p_{0}\right)}{1+\tilde{T}_{1}\left(p_{0}\right)+\tilde{T}_{2}\left(p_{0}\right)},
$$

which can be written as

$$
\tilde{F}_{\chi_{1} \chi_{1}}\left(p_{0}\right)=\frac{\tilde{H}_{1}\left(1+\tilde{T}_{2}\right)^{-2}}{\left[1+\left(1+\tilde{T}_{2}\right)^{-1} \tilde{T}_{1}\right]}+\tilde{H}_{2}\left(1+\tilde{T}_{2}\right)^{-1},
$$

or more explicitly

$$
\tilde{F}_{\chi_{1} \chi_{1}}\left(p_{0}\right)=\frac{v_{2}\left(m_{2}\right)\left(1+\tilde{T}_{2}\right)^{-2}}{\frac{\cosh m_{2}-\cos p_{0}}{\sinh m_{2}}+\left(1+\tilde{T}_{2}\right)^{-1} v_{2}\left(m_{2}\right) \tilde{K}}+\tilde{H}_{2}\left(1+\tilde{T}_{2}\right)^{-1} .
$$

Thus, the singularities of $\tilde{F}_{\chi_{1} \chi_{1}}\left(p_{0}\right)$ on $\left|\operatorname{Re} p_{0}\right| \leqq \pi, 0 \leqq \operatorname{Im} p_{0} \leqq-7(1-\varepsilon) \log \beta$ are the same as the zeros of

$$
f\left(p_{0}\right)=\frac{\cosh m_{2}-\cos p_{0}}{\sinh m_{2}}+v_{2}\left(m_{2}\right)\left(1+\tilde{T}_{2}\right)^{-1} \tilde{K}
$$

Notice that the entire function $g\left(p_{0}\right)=\frac{\cosh m_{2}-\cos p_{0}}{\sinh m_{2}}$ has only two zeros in the strip $\left|\operatorname{Re} p_{0}\right| \leqq \pi$, namely $p_{0}= \pm i m_{2}$.

Theorem 2.9. There exists $\beta_{8} \leqq \beta_{7}$ such that if $0<\beta<\beta_{8},\left|v_{2}\left(m_{2}\right)\left(1+\tilde{T}_{2}\right)^{-1} \tilde{K}\right| \leqq \frac{1}{2}$ on $\left|\operatorname{Re} p_{0}\right| \leqq \pi, 0 \leqq \operatorname{Im} p_{0} \leqq-7(1-\varepsilon) \log \beta$, and $\left|g\left(p_{0}\right)\right|>1 / 2$ on the boundary of this region.

From Rouché's theorem, it then follows that

Corollary 2.10. $\tilde{F}_{\chi_{1} \chi_{1}}\left(p_{0}\right)$ has precisely one simple pole on $\left|\operatorname{Re} p_{0}\right|<\pi, 0<\operatorname{Im} p_{0}$ $<-7(1-\varepsilon) \log \beta$, if $0<\beta<\beta_{8}$.

From (2.15), the pole of $\tilde{F}_{\chi_{1} \chi_{1}}\left(p_{0}\right)$ can lie only on the imaginary axis because, as follows from the arguments in [9], $\tilde{\Gamma}_{\chi \chi}\left(p_{0}\right)$ for $\left|\operatorname{Re} p_{0}\right| \leqq \pi$ is regular outside that axis. We denote the pole by $p_{0}=i \varrho$.

Theorem 2.11. There are constants $\zeta$ and $\beta_{9} \leqq \beta_{8}$ such that if $0<\beta<\beta_{9}, \mid v_{2}\left(m_{2}\right)$ $\left(1+\tilde{T}_{2}\right)^{-1} \tilde{K}|<| g \mid$ on the circle $\left|p_{0}-i m_{2}\right|=\zeta \beta$.

Corollary 2.12. If $0<\beta<\beta_{9},\left|m_{2}-\varrho\right|<\zeta \beta$.

As remarked earlier, $p_{0}=i \varrho$ is expected to be a pole of $\tilde{\Gamma}_{\chi \chi}\left(p_{0}\right)$. To show that this is indeed the case, define

and

$$
\tilde{L}_{\chi \chi_{1}}\left(p_{0}\right)=\tilde{\Gamma}_{\chi \chi}\left(p_{0}\right) \tilde{G}_{\chi \chi_{1}}\left(p_{0}\right) \tilde{\Phi}_{\chi_{1} \chi_{1}}\left(p_{0}\right)
$$

$$
\tilde{L}_{\chi_{1} \chi}\left(p_{0}\right)=\tilde{\Phi}_{\chi_{1} \chi_{1}}\left(p_{0}\right) \tilde{G}_{\chi_{1} \chi}\left(p_{0}\right) \tilde{\Gamma}_{\chi \chi}\left(p_{0}\right)
$$

These two functions are actually the same because in general $\hat{G}_{\varphi \psi}\left(x_{0}\right)=\overline{\hat{G}_{\psi \varphi}\left(x_{0}\right)}$ and $\chi, \chi_{1}$ are real functions. 
Theorem 2.13. There exists a constant $k_{6}$ such that if $0<\beta<\beta_{7}, \tilde{L}_{\chi \chi_{1}}\left(p_{0}\right)$ is analytic on $\left|\operatorname{Re} p_{0}\right| \leqq \pi, 0 \leqq \operatorname{Im} p_{0} \leqq-7(1-\varepsilon) \log \beta$ and $\left|\tilde{L}_{\chi x_{1}}\left(p_{0}\right)\right| \leqq k_{6} \beta^{\frac{7}{2} \varepsilon}$ there.

Define $\tilde{M}\left(p_{0}\right)$ by the equation

$$
\tilde{\Gamma}_{\chi \chi}\left(p_{0}\right)=\tilde{L}_{\chi \chi_{1}}\left(p_{0}\right) \tilde{F}_{\chi_{1} \chi_{1}}\left(p_{0}\right) \tilde{L}_{\chi_{1} \chi}\left(p_{0}\right)+\tilde{M}\left(p_{0}\right) .
$$

Theorem 2.14. $\tilde{M}\left(p_{0}\right)$ is analytic on $\left|\operatorname{Re} p_{0}\right| \leqq \pi, 0 \leqq \operatorname{Im} p_{0} \leqq-7(1-\varepsilon) \log \beta$ if $0<\beta$ $<\beta_{7}$.

From this last theorem and (2.22), we see that the only possible singularity on $\left|\operatorname{Re} p_{0}\right| \leqq \pi, 0 \leqq \operatorname{Im} p_{0} \leqq-7(1-\varepsilon) \log \beta$ of $\tilde{\Gamma}_{\chi x}\left(p_{0}\right)$ is $p_{0}=i \varrho$. To show that this is indeed a singularity, we must check that $L_{\chi \chi_{1}}(i \varrho) \neq 0$.

Theorem 2.15. There exists $\beta_{10} \leqq \beta_{9}$ such that for $0<\beta<\beta_{10}, \tilde{L}_{\chi \chi_{1}}\left(p_{0}\right) \neq 0$ if $\mid p_{0}$ $-i m_{2} \mid \leqq 1$.

We are now in position to find the zeros of $\tilde{\Gamma}_{\chi \chi}\left(p_{0}\right)$. Inserting (2.18), (2.19) into (2.22), we get

$\tilde{\Gamma}_{\chi \chi}=\frac{1}{f}\left[v_{2}\left(m_{2}\right) \tilde{L}_{\chi \chi_{1}}\left(1+\tilde{T}_{2}\right)^{-2} \tilde{L}_{\chi_{1} \chi}+\left(\tilde{L}_{\chi \chi_{1}} \tilde{H}_{2}\left(1+\tilde{T}_{2}\right)^{-1} \tilde{E}_{\chi_{1 \chi}}+\tilde{M}\right) f\right]$.

From Theorem 2.6 we know that $\tilde{\Gamma}_{\chi \chi}\left(i m_{0}\right)=0$ and this is the only zero on $0 \leqq \operatorname{Im} p_{0}$ $\leqq-6(1-\varepsilon) \log \beta$. Thus, to find new zeros there is no loss of generality in restricting $p_{0}$ to $\left|\operatorname{Re} p_{0}\right| \leqq \pi,-5 \log \beta \leqq \operatorname{Im} p_{0} \leqq-7(1-\varepsilon) \log \beta$.

Theorem 2.16. There are constants $k_{7}, \beta_{11} \leqq \beta_{10}$ such that if $0<\beta<\beta_{11}$ and $\left|\operatorname{Re} p_{0}\right|$ $\leqq \pi,-5 \log \beta \leqq \operatorname{Im} p_{0} \leqq-7(1-\varepsilon) \log \beta$,

$$
\left|\tilde{M}+\tilde{L}_{\chi \chi_{1}} \tilde{H}_{2}\left(1+\tilde{T}_{2}\right)^{-1} \tilde{L}_{\chi_{1} \chi}\right| \geqq\left(k_{7} / \beta\right) .
$$

Thus, the zeros of $\tilde{\Gamma}_{\chi \chi}$ in the region of Theorem 2.16 are the same as those for the function

$$
f_{1}=f+\frac{v_{2}\left(m_{2}\right) \tilde{L}_{\chi \chi_{1}}\left(1+\tilde{T}_{2}\right)^{-2} \tilde{L}_{\chi_{1} \chi}}{\left(\tilde{L}_{\chi \chi_{1}} \tilde{H}_{2}\left(1+\tilde{T}_{2}\right)^{-1} \tilde{L}_{\chi_{1} \chi}+\tilde{M}\right)}=g+h
$$

with $f$ and $g\left(p_{0}\right)$ as before [see definition after Eq. (2.19)] and

$$
h\left(p_{0}\right)=v_{2}\left(m_{2}\right)\left(1+\tilde{T}_{2}\right)^{-1} \tilde{K}+\frac{v_{2}\left(m_{2}\right) \tilde{L}_{\chi \chi_{1}}\left(1+\tilde{T}_{2}\right)^{-2} \tilde{L}_{\chi_{1} \chi}}{\tilde{L}_{\chi_{1} 1} \tilde{H}_{2}\left(1+\tilde{T}_{2}\right)^{-1} \tilde{L}_{\chi_{1} \chi}+\tilde{M}} .
$$

Theorem 2.17. There exists $\beta_{12} \leqq \beta_{11}$ such that for $0<\beta<\beta_{12},\left|h\left(p_{0}\right)\right| \leqq 1 / 2$ on $\left|\operatorname{Re} p_{0}\right| \leqq \pi,-5 \log \beta \leqq \operatorname{Im} p_{0} \leqq-7(1-\varepsilon) \log \beta$ and $\left|g\left(p_{0}\right)\right|>1 / 2$ on the boundary of the same region.

Because of (2.11), we know that $g\left(p_{0}\right)$ has precisely one zero in the interior of the region above. Therefore, from Rouchés theorem, the same is true for $\tilde{\Gamma}_{\chi \chi}\left(p_{0}\right)$. Call this zero $p_{0}=i m_{1}$. We have a result analogous to Theorem 2.11 .

Theorem 2.18. There are constants $\eta$ and $\beta_{13} \leqq \beta_{12}$ such that if $0<\beta<\beta_{13},\left|h\left(p_{0}\right)\right|$ $<\left|g\left(p_{0}\right)\right|$ on $\left|p_{0}-i m_{2}\right|=\eta \beta$. 
Corollary 2.19. If $0<\beta<\beta_{13},\left|m_{1}-m_{2}\right|<\eta \beta$.

The theorems above prove that on $\left|\operatorname{Re} p_{0}\right| \leqq \pi, 0 \leqq \operatorname{Im} p_{0}<-7(1-\varepsilon) \log \beta$, $\tilde{G}_{x x}\left(p_{0}\right)$ has precisely two simple poles, at $p_{0}=i m_{0}$ and $p_{0}=i m_{1}$. The next result shows that this is also the case for $\tilde{G}_{\chi_{1} \chi_{1}}$ :

Theorem 2.20. There exists $\beta_{14} \leqq \beta_{13}$ such that if $0<\beta<\beta_{14}, \tilde{G}_{x_{1} x_{1}}\left(p_{0}\right)$ has exactly two simple poles on $\left|\operatorname{Re} p_{0}\right| \leqq \pi, 0 \leqq \operatorname{Im} p_{0}<-7(1-\varepsilon) \log \beta$, namely $p_{0}=i m_{0}$ and $p_{0}$ $=i m_{1}$.

Finally, we extend this result to arbitrary $\tilde{G}_{\varphi_{1} \varphi_{1}}$ :

Theorem 2.21. If $0<\beta<\beta_{14}$ and $\varphi=P_{\mathbf{a}}^{(1)} \varphi$ for some $\mathbf{a}, \tilde{G}_{\varphi \varphi}\left(p_{0}\right)$ is analytic on $\left|\operatorname{Re} p_{0}\right|$ $\leqq \pi, 0 \leqq \operatorname{Im} p_{0}<-7(1-\varepsilon) \log \beta$ except possibly for simple poles at $p_{0}=i m_{0}, p_{0}$ $=i m_{2}$.

\section{Properties of Finite Volume Generalized Correlation Functions}

The aim of this section is to calculate derivatives of

$$
G_{\varphi \psi}\left(x, y ; \Lambda ;\left\{\beta_{P}\right\}\right)=\langle\bar{\varphi}(x) \psi(y)\rangle\left(\Lambda ;\left\{\beta_{P}\right\}\right)-\langle\bar{\varphi}(x)\rangle\langle\psi(y)\rangle
$$

with respect to $\beta_{P}$ for a suitable choice of these variables. See the beginning of Sect. 2 for notation. This will be the main technical input to determine the domains of analyticity of the functions introduced in the last section. As usual, we assume $\varphi$, $\psi$ of finite support in the time zero plane and e.g. $\varphi(x)$ denotes the translate of $\varphi$ by the lattice vector $x=\left(x_{0}, \mathbf{x}\right)$. We also assume the space-time dimensionality to be three and that the character $\chi$ in (2.1) is real irreducible. This assumption is responsible for the simple forms of Theorems 3.2 and 3.3 below. We use periodic boundary conditions in the space directions only, and without loss of generality, assume the region $\Lambda \subset \mathbb{Z}^{3}$ to be a rectangle along the coordinate axis. Setting all $\beta_{P}$ equal to small $\beta$, the thermodynamic limit of (3.1) does not depend on this particular choice of boundary conditions, as follows easily from the cluster expansion [8].

The specific choice of $\left\{\beta_{P}\right\}$ variables we will adopt is the following. We set $\beta_{P}=w_{q}$ for all plaquettes $P$ parallel to the time axis and located between $t=q$ and $t=q+1$. For plaquettes perpendicular to the time axis, we set $\beta_{P}=z$. In terms of duplicate variables, (3.1) can be written as

$$
G_{\varphi \psi}(x, y ; \Lambda)=\frac{1}{2 Z_{\Lambda}^{2}} \iint\left(\bar{\varphi}(x)-\bar{\varphi}^{\prime}(x)\right)\left(\psi(y)-\psi^{\prime}(y)\right) e^{S_{\Lambda}\left(g, g^{\prime}\right)} d g_{\Lambda} d g_{\Lambda}^{\prime} \equiv \frac{\mathscr{S}}{2 \mathscr{D}},
$$

where e.g. $\varphi^{\prime}$ is $\varphi$ at $g^{\prime}$ and

$$
S_{\Lambda}\left(g, g^{\prime}\right)=\sum_{q} w_{q} \sum_{P \in \mathscr{P}_{q}^{\prime \prime}}\left(\chi\left(g_{P}\right)+\chi\left(g_{P}^{\prime}\right)\right)+z \sum_{P \in \mathscr{P}\lrcorner}\left(\chi\left(g_{P}\right)+\chi\left(g_{P}^{\prime}\right)\right) .
$$

The sum over $q$ is only for times defined within $\Lambda, \mathscr{P}_{q}^{\prime \prime}$ is the set of plaquettes in $\Lambda$ parallel to the time direction and located between the time layers $t=q, t=q+1$, and $\mathscr{P}^{\perp}$ is the set of plaquettes in $\Lambda$ perpendicular to the time direction. Also,

$$
Z_{\Lambda}^{2}=\mathscr{D}=\iint e^{S_{\Lambda}\left(g, g^{\prime}\right)} d g_{\Lambda} d g_{\Lambda}^{\prime} .
$$


We want to find the structure of the coefficients of the power series expansion of (3.2) in a particular variable $w_{q}$. This has been done in $[5,6]$ up to the coefficient of fifth order. For the present work, we need the coefficient of the sixth order as well.

Write (3.3) in the form

$$
S_{\Lambda}\left(g, g^{\prime}\right)=w_{q} \sum_{P \in \mathscr{P}_{q}^{\prime \prime}}\left(\chi\left(g_{P}\right)+\chi\left(g_{P}^{\prime}\right)\right)+S_{\Lambda}^{(q)}\left(g, g^{\prime}\right),
$$

and let $G(\Lambda)$ be the matrix whose elements are $G(x, y ; \Lambda)$.

We will always assume $|z|,\left|w_{q}\right|<\beta_{0}$ so that the results of the cluster expansion applies to $G_{\varphi \psi}(x, y ; \Lambda)$.

\section{Theorem 3.1.}

(a) $\left.\left(\partial^{m} / \partial w_{q}^{m}\right)\right|_{w_{q}=0} G(\Lambda)=0$ if $1 \leqq m \leqq 3$,

(b) $\left.\left(\partial^{m} / \partial w_{q}^{m}\right)\right|_{w_{q}=0} ^{w_{q}} G(x, y ; \Lambda)=0$ if $x_{0} \leqq q<y_{0}$ and $0 \leqq m \leqq 3$.

Proof. The numerator $\mathscr{S}$ of (3.2) has the expansion

with

$$
\mathscr{S}=\sum_{n=0}^{\infty} \frac{1}{n !} \mathscr{S}_{n}^{(q)}(x, y) w_{q}^{n},
$$

$$
\mathscr{S}_{n}^{(q)}(x, y)=\iint(\bar{\varphi}(x)-\bar{\varphi}(x))\left(\psi(y)-\psi^{\prime}(y)\right) e_{\Lambda}^{S(q)\left(g, g^{\prime}\right)}\left(\sum_{\boldsymbol{P} \in \mathscr{P}_{q}^{\prime \prime}}\left(\chi\left(g_{P}\right)+\chi\left(g_{P}^{\prime}\right)\right)\right)^{n} d g_{\Lambda} d g_{\Lambda}^{\prime} .
$$

To calculate (3.7) we use the Peter-Weyl orthogonality theorem together with the fact that $\left(\bar{\varphi}(x)-\bar{\varphi}^{\prime}(x)\right)\left(\psi(y)-\psi^{\prime}(y)\right) \exp \left(S_{\Lambda}^{(q)}\left(g, g^{\prime}\right)\right)$ do not depend on the bond variables parallel to the time direction and located between the time layers $t=q$, $t=q+1$. Thus, $\mathscr{S}_{1}^{(q)}(x, y)=0$. When $n=2$, it is clear that

$$
\begin{aligned}
\mathscr{S}_{2}^{(q)}(x, y) & =\sum_{P \in \mathscr{P}_{q}^{\prime \prime}} \iint\left(\bar{\varphi}(x)-\bar{\varphi}^{\prime}(x)\right)\left(\psi(y)-\psi^{\prime}(y)\right) e_{\Lambda}^{S(q)}\left(\chi\left(g_{P}\right)^{2}+\chi\left(g_{P}^{\prime}\right)^{2}\right) d g_{\Lambda} d g_{\Lambda}^{\prime} \\
& =\sum_{P \in \mathscr{P}_{q}^{\prime \prime}} 2 \mathscr{S}_{0}^{(q)}(x, y)=2 \mathscr{A}(\Lambda) \mathscr{P}_{0}^{(q)}(x, y),
\end{aligned}
$$

where $\mathscr{A}(\Lambda)$ is the number of plaquettes in $\mathscr{P}_{q}^{\prime \prime}$ (which is just four times the area of a time layer). Similarly, when $n=3$, the only terms giving a nonzero contribution in the expansion of the third power in (3.7) are $\sum_{P} \chi\left(g_{P}\right)^{3}$ and $\sum_{P} \chi\left(g_{P}^{\prime}\right)^{3}$. Therefore,

$$
\mathscr{S}_{3}^{(q)}(x, y)=2 \alpha \mathscr{A}(\Lambda) \mathscr{S}_{0}^{(q)}(x, y),
$$

where $\alpha=\int \chi(g)^{3} d g$. The denominator in (3.2) has of course a similar expansion:

with

$$
\mathscr{D}=\sum_{n=0}^{\infty} \frac{1}{n !} \mathscr{D}_{n}^{(q)} w_{q}^{n}
$$

$$
\mathscr{D}_{1}^{(q)}=0, \quad \mathscr{D}_{2}^{(q)}=2 \mathscr{A}(\Lambda) \mathscr{D}_{0}^{(q)} \quad \text { and } \quad \mathscr{D}_{3}^{(q)}=2 \alpha \mathscr{A}(\Lambda) \mathscr{D}_{0}^{(q)} .
$$

From this, it follows immediately that

$$
G_{\varphi \psi}(x, y ; \Lambda)=\left.G_{\varphi \psi}(x, y ; \Lambda)\right|_{w_{q}=0}+O\left(w_{q}^{4}\right),
$$

which proves part (a) of the theorem. Part (b) also follows from (3.12), since if $x_{0} \leqq q \leqq y_{0}$, then clearly $\left.G_{\varphi \varphi}(x, y ; \Lambda)\right|_{w_{q}=0}=0$. 
The next theorem was established in $[5,6]$. Its simple proof is reproduced here for completeness. Let $\hat{e}_{0}$ denote the unit vector in the time direction.

Theorem 3.2. Assume $x_{0} \leqq q \leqq y_{0}$. There are constants $c_{4}>0$ and $c_{5}$ such that $(m=4,5)$

$$
\left.\frac{\partial^{m}}{\partial w_{q}^{m}} G_{\varphi \psi}(x, y ; \Lambda)\right|_{w_{q}=0}=\left.c_{m} \sum_{t_{0}=q} G_{\varphi \chi}(x, t ; \Lambda) G_{\chi \psi}\left(t+\hat{e}_{0}, y ; \Lambda\right)\right|_{w_{q}=0} .
$$

Proof. Since $\mathscr{S}_{n}^{(q)}(x, y)=0$ from $0 \leqq n \leqq 3$, we have $(m=4,5)$

$$
\left.\frac{\partial^{m}}{\partial w_{2}^{m}} G_{\varphi \psi}(x, y ; \Lambda)\right|_{w_{q}=0}=\frac{1}{2} \frac{\mathscr{S}_{m}^{(q)}(x, y)}{\mathscr{D}_{0}^{(q)}} \text {. }
$$

Expanding the fourth power in (3.7) with $n=4$, one shows that the only nonvanishing contributions come from terms of the form $\prod_{i=1}^{4} \chi\left(g_{P_{i}}\right)$ or $\prod_{i=1}^{4} \chi\left(g_{P_{i}}^{\prime}\right)$ with the plaquettes $\left\{P_{i}\right\}_{i=1}^{4}$ disposed along the faces of cube. In this case, the product of characters can be integrated explicitly, yielding

$$
\int \prod_{i=1}^{4} \chi\left(g_{P_{i}}\right) \prod_{\ell=1}^{4} d g_{\ell}=\frac{1}{d^{4}} \chi\left(g_{P_{5}}\right) \chi\left(g_{P_{6}}\right) \text {. }
$$

The integration above is performed only with respect to bond variables parallel to the time direction, and $P_{5}, P_{6}$ are the remaining faces (which are perpendicular to the time direction) of the cube under consideration; $d$ is the dimension of the representation defining $\chi$. Thus, $\mathscr{S}_{4}^{(q)}(x, y)$ has the form

$$
\begin{aligned}
& \mathscr{S}_{4}^{(q)}(x, y)=c_{4} \sum_{t_{0}=q} \iint\left(\bar{\varphi}(x)-\bar{\varphi}^{\prime}(x)\right)\left(\psi(y)-\psi^{\prime}(y)\right) \\
& \cdot e^{S_{A}^{(q)}}\left(\chi(t) \chi\left(t+\hat{\ell}_{0}\right)+\chi^{\prime}(t) \chi^{\prime}\left(t+\hat{\ell}_{0}\right)\right) d g_{\Lambda} d g_{\Lambda}^{\prime},
\end{aligned}
$$

where $c_{4}$ is just a combinatorial factor [divided by $d^{4}$ from (3.13)], which counts the number of terms arising from (3.7) associated to a fixed elementary cube in the time slice $t=q, t=q+1$. This proves the theorem when $m=4$.

When $m=5$ we proceed as above with $\mathscr{S}_{5}^{(q)}(x, y)$. Expanding the fifth power in (3.7), nonvanishing contributions come only from terms like $\prod_{i=1}^{5} \chi\left(g_{P_{i}}\right)$ or $\prod_{i=1}^{5} \chi\left(g_{P_{i}}^{\prime}\right)$ with the five plaquettes disposed along the faces of a cube. The side carrying a $\chi^{2}$ term can be expanded in a Fourier series and the only term in this expansion giving a non-zero contribution to the integral $\int \prod_{i=1}^{5} \chi\left(g_{P_{i}}\right) \prod_{\ell=1}^{4} d g_{\ell}$ comes from the component along $\chi$. Thus, the result is proportional to (3.13) and the theorem is proved also for $m=5$.

The sixth derivative of $G_{\varphi \psi}(x, y ; \Lambda)$ has a richer structure:

Theorem 3.3. There are constants $c_{6}, c_{7}\left(c_{7}>0\right)$ such that if $x_{0} \leqq q<y_{0}$, then

$$
\begin{aligned}
\left.\frac{\partial^{6}}{\partial w_{q}^{6}} G_{\varphi \psi}(x, y ; \Lambda)\right|_{w_{q}=0}= & \left.c_{6} \sum_{t_{0}=q} G_{\varphi \chi}(x, t ; \Lambda) G_{\chi \psi}\left(t+\hat{e}_{0}, y ; \Lambda\right)\right|_{w_{q}=0} \\
& +\left.c_{7} \sum_{i=1}^{4} \sum_{t_{0}=q} G_{\varphi \chi_{i}}(x, t ; \Lambda) G_{\chi_{i} \psi}\left(t+\hat{e}_{0}, y ; \Lambda\right)\right|_{w_{q}=0},
\end{aligned}
$$

where $\chi_{i}=P_{0}^{(i)} \chi_{h}, 1 \leqq i \leqq 4$ were defined in Sect. 2 . 
Proof. We have

$$
\left.\frac{\partial^{6}}{\partial w_{q}^{6}} G_{\varphi \psi}(x, y ; \Lambda)\right|_{w_{q}=0}=\frac{\mathscr{S}_{6}^{(q)}(x, y)}{2 \mathscr{D}_{0}^{(q)}}-\frac{6 !}{4 !} \mathscr{A}(\Lambda) \frac{\mathscr{S}_{4}^{(q)}(x, y)}{2 \mathscr{D}_{0}^{(q)}},
$$

with

$$
\begin{aligned}
\mathscr{S}_{6}^{(q)}(x, y)= & \sum_{P_{\imath} \in \mathscr{P}_{q}^{\prime \prime}} \iint\left(\bar{\varphi}(x)-\bar{\varphi}^{\prime}(x)\right)\left(\psi(y)-\psi^{\prime}(y)\right) \\
& \cdot e_{\Lambda}^{S_{\Lambda}^{(q)}} \prod_{i=1}^{6}\left(\chi\left(g_{P_{i}}\right)+\chi\left(g_{P_{\imath}}^{\prime}\right)\right) d g_{\Lambda} d g_{\Lambda}^{\prime} .
\end{aligned}
$$

Expanding the product we see that cross terms of the form $\chi^{2} \chi^{\prime 4}$ or $\chi^{4} \chi^{\prime 2}$ give nonvanishing contributions proportional to $\mathscr{A}(\Lambda) \mathscr{S}_{4}^{(q)}(x, y) /\left(2 \mathscr{D}_{0}^{(q)}\right)$. Also the "pure" terms $\chi^{6}, \chi^{\prime 6}$ have contributions proportional to $\mathscr{A}(\Lambda) \mathscr{S}_{4}^{(q)}(x, y) /\left(2 \mathscr{D}_{0}^{(q)}\right)$. This happens when the term is of the form $\chi^{2}\left(g_{P}\right) \prod_{i=1}^{4} \chi\left(g_{P_{i}}\right)$ with $\left\{P_{i}\right\}$ disposed along the faces of a cube and $P$ different from the $P_{i}$ 's. It is not difficult to show that the terms proportional to $\mathscr{A}(\Lambda) \mathscr{S}_{4}^{(q)}(x, y) /\left(2 \mathscr{D}_{0}^{(q)}\right)$ add up to zero. This is to be expected, since from the cluster expansion, $G_{\varphi \psi}\left(x, y ; \Lambda ;\left\{w_{q}\right\}, z\right)$ has a limit as $\Lambda \rightarrow \mathbb{Z}^{3}$, which is analytic in each variable (the limit is attained uniformly on compact subsets of $\left|w_{q}\right|$, $\left.|z|<\beta_{0}\right)$. Then $\left.\lim _{\Lambda \rightarrow \mathbb{Z}^{3}}\left(\partial^{6} / \partial w_{q}^{6}\right)\right|_{w_{q}=0} G_{\varphi \psi}(x, y ; \Lambda)$ exists, showing that extensive terms must cancel in the expression for the sixth derivative. There are still contributions proportional to $\mathscr{S}_{4}^{(q)} /\left(2 \mathscr{D}_{0}^{(q)}\right)$. This happens when all six plaquettes in $\prod_{i=1}^{6} \chi\left(g_{P_{i}}\right)$ are disposed along the sides of a cube. We then proceed as in the $\mathscr{S}_{5}^{(q)}$ case by Fourier transforming the plaquettes containing $\chi$ with a power higher than one. The sum of all these terms gives a contribution to $\left.\left(\partial^{6} / \partial w_{q}^{6}\right)\right|_{w_{q}=0} G_{\varphi \psi}(x, y ; \Lambda)$ of the form $\left.c_{6} \sum_{t_{0}=q} G_{\varphi \chi}(x, t ; \Lambda) G_{\chi \psi}\left(t+\hat{e}_{0}, y ; \Lambda\right)\right|_{w_{q}=0}$ for a suitable constant $c_{6}$.

The other contribution from $\mathscr{S}_{6}^{(q)}(x, y)$ come when the six plaquettes in $\prod_{i=1}^{6}$ $\prod_{1} \chi\left(g_{P_{l}}\right)$ are disposed along the faces of a parallelepiped. The calculation of such terms is similar to the one corresponding to $\mathscr{S}_{4}^{(q)}(x, y)$. Let $\chi_{h}$ and $\chi_{v}$ be the horizontal and vertical windows at the origin in the time zero plane. $\chi_{h}$ was defined after Theorem 2.1, and $\chi_{v}=R_{0} \chi_{h}$. The contribution of these terms to $\mathscr{S}_{6}^{(q)}$ is given by

$$
\begin{aligned}
& c_{7}^{\prime} \sum_{t_{0}=q} \iint\left(\bar{\varphi}(x)-\bar{\varphi}^{\prime}(x)\right)\left(\psi(y)-\psi^{\prime}(y)\right) e_{\Lambda}^{S(q)}\left(\chi_{h}(t) \chi_{h}\left(t+\hat{e}_{0}\right)+\chi_{v}(t) \chi_{v}\left(t+\hat{e}_{0}\right)\right. \\
& \left.\quad+\chi_{h}^{\prime}(t) \chi_{h}^{\prime}\left(t+\hat{e}_{0}\right)+\chi_{v}^{\prime}(t) \chi_{v}^{\prime}\left(t+\hat{e}_{0}\right)\right) d g_{\Lambda} d g_{\Lambda}^{\prime},
\end{aligned}
$$

where $c_{7}^{\prime}$ is a combinatorial factor (divided by $d^{6}$ ) analogous to $c_{4}$ and therefore positive. Thus, the final expression for the sixth derivative is

$$
\begin{aligned}
& \left.\frac{\partial^{6}}{\partial w_{q}^{6}} G_{\varphi \psi}(x, y ; \Lambda)\right|_{w_{q}=0}=\left.c_{6} \sum_{t_{0}=q} G_{\varphi \chi}(x, t ; \Lambda) G_{\chi \psi}\left(t+\hat{e}_{0}, y ; \Lambda\right)\right|_{w_{q}=0} \\
& \quad+\left.c_{7}^{\prime} \sum_{t_{0}=q}\left[G_{\varphi \chi_{h}}(x, t ; \Lambda) G_{\chi_{h} \psi}\left(t+\hat{e}_{0}, y ; \Lambda\right)+G_{\varphi \chi_{v}}(x, t ; \Lambda) G_{\chi_{v} \psi}\left(t+\hat{e}_{0}, y ; \Lambda\right)\right]\right|_{w_{q}=0} .
\end{aligned}
$$


This can be reexpressed in terms of $\chi_{i}, 1 \leqq i \leqq 4$ by using the identity

$$
\begin{aligned}
& \sum_{i=1}^{4} G_{\varphi \chi_{2}}(x, t ; \Lambda) G_{\chi_{i} \psi}\left(t+\hat{e}_{0}, y ; \Lambda\right)=\frac{1}{4}\left[G_{\varphi \chi_{h}}(x, t ; \Lambda) G_{\chi_{h} \psi}\left(t+\hat{e}_{0}, y ; \Lambda\right)\right. \\
& \quad+G_{\varphi \chi_{v}}(x, t ; \Lambda) G_{\chi_{v}}\left(t+\hat{e}_{0}, y ; \Lambda\right)+G_{\varphi \chi_{h}}\left(x, t-\hat{e}_{1} ; \Lambda\right) G_{\chi_{h} \psi}\left(t-\hat{e}_{1}+\hat{e}_{0}, y ; \Lambda\right) \\
& \left.\quad+G_{\varphi \chi_{v}}\left(x, t-\hat{e}_{2} ; \Lambda\right) G_{\chi_{v} \psi}\left(t-\hat{e}_{2}+\hat{e}_{0}, y ; \Lambda\right)\right],
\end{aligned}
$$

where $\hat{e}_{1}, \hat{e}_{2}$ are the unit vectors along the horizontal and vertical directions, respectively. Using the periodicity in the space directions and setting $c_{7}=2 c_{7}^{\prime}$ completes the proof of the theorem.

The partial Fourier transform (with respect to the space variables) at zero momentum is defined by

$$
\hat{G}_{\varphi \psi}\left(x_{0}, y_{0} ; \Lambda\right)=\sum_{\mathbf{y}} G_{\varphi \psi}(x, y ; \Lambda),
$$

the sum being clearly independent of $\mathbf{x}$ due to spatial translation invariance of the finite volume interacting measure (with complex parameters $\left.\left\{w_{q}\right\}, z\right)$. Let $\hat{G}_{\varphi \psi}(\Lambda)$ be the matrix whose elements are $\hat{G}_{\varphi \psi}\left(x_{0}, y_{0} ; \Lambda\right)$. We summarize the results obtained in this section in the following

\section{Theorem 3.4.}

(a) $\left.\left(\partial^{m} / \partial w_{q}^{m}\right)\right|_{w_{q}=0} \hat{G}_{\varphi \psi}(\Lambda)=0$ if $1 \leqq m \leqq 3$.

(b) If $x_{0} \leqq q<y_{0}$, then

$$
\left.\left(\partial^{m} / \partial w_{q}^{m}\right)\right|_{w_{q}=0} \hat{G}_{\varphi \psi}\left(x_{0}, y_{0} ; \Lambda\right)=0, \quad 0 \leqq m \leqq 3 .
$$

(c) There are constants $c_{4}>0$ and $c_{5}$ such that if $x_{0} \leqq q<y_{0}$, then

$$
\left.\left(\partial^{m} / \partial w_{q}^{m}\right)\right|_{w_{q}=0} \hat{G}_{\varphi \psi}\left(x_{0}, y_{0} ; \Lambda\right)=\left.c_{m} \hat{G}_{\varphi x}\left(x_{0}, q ; \Lambda\right) \hat{G}_{\chi \psi}\left(q+1, y_{0} ; \Lambda\right)\right|_{w_{q}=0}, \quad m=4,5 .
$$

(d) There are constants $c_{6}, c_{7}>0$ such that for $x_{0} \leqq q<y_{0}$,

$$
\begin{aligned}
\left.\frac{\partial^{6}}{\partial w_{q}^{6}} \hat{G}_{\varphi \psi}\left(x_{0}, y_{0} ; \Lambda\right)\right|_{w_{q}=0}= & \left.c_{6} \hat{G}_{\varphi \chi}\left(x_{0}, q ; \Lambda\right) \hat{G}_{\chi \psi}\left(q+1, y_{0} ; \Lambda\right)\right|_{w_{q}=0} \\
& +\left.c_{7} \sum_{i=1}^{2} \hat{G}_{\varphi \chi_{2}}\left(x_{0}, q ; \Lambda\right) \hat{G}_{\chi_{\imath} \psi}\left(q+1, y_{0} ; \Lambda\right)\right|_{w_{q}=0} .
\end{aligned}
$$

Proof. We have only to explain why there is no contribution due to $\chi_{i}, i=3,4$ in the last sum in (d). This is because, e.g.

$$
\begin{aligned}
\hat{G}_{\varphi \chi_{3}}\left(x_{0}, y_{0} ; \Lambda\right)= & \sum_{\mathbf{y}} G_{\varphi \chi_{3}}(x, y ; \Lambda)=\frac{1}{4} \sum_{\mathbf{y}}\left[G_{\varphi \chi_{h}}(x, y ; \Lambda)\right. \\
& \left.+i G_{\varphi \chi_{v}}(x, y ; \Lambda)-G_{\varphi \chi_{h}}\left(x, y-\hat{e}_{1} ; \Lambda\right)-i G_{\varphi \chi_{v}}\left(x, y-\hat{e}_{2} ; \Lambda\right)\right]=0 .
\end{aligned}
$$

\section{Proof of Theorems in Sect. 2}

In this section, the missing proofs of the results stated in Sect. 2 will be presented. We start with the observation that an equation analogous to (2.9) holds even for finite volume correlation functions depending on the complex parameters $\left\{w_{q}\right\}, z$. 
Namely,

$$
G_{\varphi \psi}\left(\left(x_{0}, \mathbf{x}\right),\left(y_{0}, \mathbf{y}\right) ; \Lambda\right)=G_{R_{\mathbf{a}} \varphi, R_{\mathbf{a}} \psi}\left(\left(x_{0}, R_{\mathbf{a}} \mathbf{x}\right),\left(y_{0}, R_{\mathbf{a}} \mathbf{y}\right) ; \Lambda\right) .
$$

The reason is that the finite volume interacting measure (with complex parameters $\left.\left\{w_{q}\right\}, z\right)$ is invariant under a rotation of $\pi / 2$ around an axis parallel to the time direction and through the center of a plaquette. Thus, the finite volume partial Fourier transforms (3.15) satisfy $\hat{G}_{\varphi \psi}\left(x_{0}, y_{0} ; \Lambda\right)=\hat{G}_{R_{\mathrm{a}} \varphi, R_{\mathrm{a}} \psi}\left(x_{0}, y_{0} ; \Lambda\right)$ and by the same argument as in Sect. 2,

$$
\hat{G}_{\varphi \psi}\left(x_{0}, y_{0} ; \Lambda\right)=\hat{G}_{R_{\mathbf{a}} \varphi, R_{\mathbf{b}} \psi}\left(x_{0}, y_{0} ; \Lambda\right)
$$

for arbitrary a, b. Also, from the cluster expansion, if $\left|w_{q}\right|,|z|<\beta_{0}, \sum_{y}\left|G_{\varphi \psi}(x, y ; \Lambda)\right|$ is bounded independent of $x$ and $\Lambda$. We call this bound $C_{\varphi \psi}[(2.7)$ thus follows from this fact]. Setting all $w_{q}, z$ equal to the same $\beta, 0<\beta<\beta_{0}$ we have $\lim _{\Lambda \rightarrow \mathbb{Z}^{3}} \hat{G}_{\varphi \psi}\left(x_{0}, y_{0} ; \Lambda\right)=\hat{G}_{\varphi \psi}\left(y_{0}-x_{0}\right)$ defined by (2.4).

Proof of Theorem 2.1. If $\varphi=P_{\mathbf{a}}^{(i)} \varphi$ with $i=3$ or 4 , then $\hat{G}_{\chi \varphi}\left(x_{0}, y_{0} ; \Lambda\right)$ $=G_{\varphi x_{i}}\left(x_{0}, y_{0} ; \Lambda\right)=0$ for $i=1,2$. Hence, from Theorem 3.4, if $x_{0} \leqq q<y_{0}$, $\left.\left(\partial^{m} / \partial w_{q}^{m}\right)\right|_{w_{q}=0} \hat{G}_{\varphi \varphi}\left(x_{0}, y_{0} ; \Lambda\right)=0$ for $0 \leqq m \leqq 6$. From the maximum modulus theorem, it then follows that $\left|\hat{G}_{\varphi \varphi}\left(x_{0}, y_{0} ; \Lambda\right)\right| \leqq C_{\varphi \varphi} \prod_{x_{0} \leqq q<y_{0}}\left|w_{q} / \beta_{0}\right|^{7}$. Setting all $w_{q}, z$ equal to $\beta, 0<\beta<\beta_{0}$ and letting $\Lambda \rightarrow \mathbb{Z}^{3}$, we get $\left|\hat{G}_{\varphi \varphi}\left(x_{0}\right)\right| \leqq C_{\varphi \varphi}\left(\beta / \beta_{0}\right)^{7\left|x_{0}\right|}$. This implies $\hat{G}_{\varphi \varphi}\left(p_{0}\right)$ to be analytic on $\left|\operatorname{Im} p_{0}\right|<-7 \log \left(\beta / \beta_{0}\right)$. Choosing $\beta<\beta_{1} \equiv \beta_{0}^{2 / \varepsilon}$ guarantees that this region contains $\left|\operatorname{Im} p_{0}\right|<-7\left(1-\frac{\varepsilon}{2}\right) \log \beta$.

Proof of Theorem 2.2. From Theorem 3.4, we have if $x_{0} \leqq q<y_{0}$ :

$$
\left.\frac{\partial^{m}}{\partial w_{q}^{m}} \hat{G}_{\chi_{2} \chi_{2}}\left(x_{0}, y_{0} ; \Lambda\right)\right|_{w_{q}=0}=0 \text { for } 0 \leqq m \leqq 5 \text {, }
$$

and

$$
\left.\frac{\partial^{6}}{\partial w_{q}^{6}} \hat{G}_{\chi_{2} \chi_{2}}\left(x_{0}, y_{0} ; \Lambda\right)\right|_{w_{q}=0}=\left.c_{7} \hat{G}_{\chi_{2} \chi_{2}}\left(x_{0}, q ; \Lambda\right) \hat{G}_{\chi_{2} \chi_{2}}\left(q+1, y_{0} ; \Lambda\right)\right|_{w_{q}=0} \text {. }
$$

As in the proof of Theorem 2.1, (4.1) implies that for $0<\beta<\beta_{0}$,

$$
\left|\hat{G}_{\chi_{2} \chi_{2}}\left(x_{0}\right)\right| \leqq C_{\chi_{2} \chi_{2}}\left(\beta / \beta_{0}\right)^{6\left|x_{0}\right|} .
$$

On the other hand, setting $x_{0}=0, y_{0}=1$ in (4.2) yields

$$
\begin{aligned}
\hat{G}_{\chi_{2} \chi_{2}}\left(0,1 ; \Lambda ;\left\{w_{q}\right\}, z\right)= & \frac{c_{7}}{6 !} \hat{G}_{\chi_{2} \chi_{2}}\left(0,0 ; \Lambda ;\left\{w_{q}\right\}_{q \neq 0}, z\right) \\
& \cdot \hat{G}_{\chi_{2} \chi_{2}}\left(1,1 ; \Lambda ;\left\{w_{q}\right\}_{q \neq 0}, z\right) w_{0}^{6}+O\left(w_{0}^{7}\right),
\end{aligned}
$$

from which we get

$$
\lim _{\beta \downarrow 0} \frac{1}{\beta^{6}} \hat{G}_{\chi_{2} \chi_{2}}(0,1 ; \Lambda ; \beta)=\frac{c_{7}}{6 !} \hat{G}_{\chi_{2} \chi_{2}}(0,0 ; \Lambda ; \beta=0) \hat{G}_{\chi_{2} \chi_{2}}(1,1 ; \Lambda ; \beta=0) .
$$


The right hand side is easily calculable, since

$$
\hat{G}_{\chi_{2} \chi_{2}}(0,0 ; \Lambda ; \beta=0)=\hat{G}_{\chi_{2} \chi_{2}}(1,1 ; \Lambda ; \beta=0)=1 / 2 .
$$

Letting $\Lambda \rightarrow \mathbb{Z}^{3}$, we conclude that

$$
\lim _{\beta \rightarrow 0} \beta^{-6} \hat{G}_{\chi_{2} \chi_{2}}\left(x_{0}=1\right)=c_{7} /(4 \cdot 6 !) .
$$

Thus, there exists $\beta_{2} \leqq \beta_{1}$ such that

$$
\hat{G}_{\chi_{2} x_{2}}\left(x_{0}=1\right) \geqq\left(c_{7} \beta^{6}\right) /(8 \cdot 6 !)
$$

if $0<\beta<\beta_{2}$. From the integral representation [arising from (2.8)],

$$
\hat{G}_{\chi_{2} \chi_{2}}\left(x_{0}\right)=\int_{(0, \infty)} e^{-\lambda_{0}\left|x_{0}\right|} d v_{2}\left(\lambda_{0}\right)
$$

(with $d v_{2}$ a positive, finite measure) and Hölder's inequality, we have $\left(x_{0} \neq 0\right)$

$$
\hat{G}_{\chi_{2} \chi_{2}}\left(x_{0}=1\right)^{\left|x_{0}\right|} \leqq \hat{G}_{\chi_{2} \chi_{2}}\left(x_{0}\right) \hat{G}_{\chi_{2} \chi_{2}}\left(x_{0}=0\right)^{\left|x_{0}\right|-1} \leqq \hat{G}_{\chi_{2} \chi_{2}}\left(x_{0}\right) C_{\chi_{2} \chi_{2}}^{\left|x_{0}\right|-1},
$$

so that

$$
\hat{G}_{\chi_{2} \chi_{2}}\left(x_{0}\right) \geqq C_{\chi_{2} \chi_{2}}\left(\frac{c_{7}}{C_{\chi_{2} \chi_{2}} \cdot 8 \cdot 6 !} \beta^{6}\right)^{\left|x_{0}\right|} \text {. }
$$

The proof of Theorem 2.2 follows from (4.3) and (4.4).

Proof of Theorem 2.3. We first show that the matrix $\hat{G}_{\chi_{2} x_{2}}(\Lambda)$ is invertible if $\left|w_{q}\right|,|z|$ are small enough. Let $P_{\chi_{2} \chi_{2}}(\Lambda)$ be the diagonal part of $G_{\chi_{2} \chi_{2}}(\Lambda)$, i.e. $P_{\chi_{2} \chi_{2}}\left(x_{0}, y_{0} ; A\right)$ $=\hat{G}_{\chi_{2} \chi_{2}}\left(x_{0}, y_{0} ; \Lambda\right) \delta_{x_{0}, y_{0}}$, and let $Q_{\chi_{2} x_{2}}(\Lambda)$ be defined by $\hat{G}_{\chi_{2 x_{2}}}(\Lambda)=P_{\chi_{2} \chi_{2}}(\Lambda)$ $+Q_{x_{2} x_{2}}(\Lambda)$. From the fundamental theorem of calculus,

$$
\hat{G}_{\chi_{2 \times 2}}\left(x_{0}, x_{0} ; \Lambda ;\left\{w_{q}\right\}, z\right)=\frac{1}{2}+\int_{0}^{1} d \lambda \frac{d}{d \lambda} \hat{G}_{\chi_{2} \chi_{2}}\left(x_{0}, x_{0} ; \Lambda ;\left\{\lambda w_{q}\right\}, \lambda z\right) .
$$

Restricting $\left\{w_{q}\right\}, z$ to $\left|w_{q}\right|,|z|<\beta_{0}^{\prime} \equiv \beta_{0} / N$ ( $N$ to be determined shorly), the derivative above can be estimated by Cauchy's formula

$$
\frac{d}{d \lambda} \hat{G}_{\chi_{2 x_{2}}}\left(x_{0}, x_{0} ; \Lambda ;\left\{\lambda w_{q}\right\}, \lambda z\right)=\frac{1}{2 \pi i} \oint_{|\eta|=N} \frac{\hat{G}_{\chi_{2} \chi_{2}}\left(x_{0}, x_{0} ; \Lambda ;\left\{\lambda w_{q}\right\}, \eta z\right)}{(\eta-\lambda)^{2}} d \eta .
$$

Since $0 \leqq \lambda \leqq 1$, we have

$$
\left|\frac{d}{d \lambda} \hat{G}_{\chi_{2 \chi_{2}}}\left(x_{0}, x_{0} ; \Lambda ;\left\{\lambda w_{q}\right\}, \lambda z\right)\right| \leqq \frac{N C_{\chi_{2} \chi_{2}}}{(N-1)^{2}} .
$$

We choose $N>1$ to be the smallest number for which the right-hand side above is less than or equal to $1 / 4$. This implies $\left\|P_{\chi_{2} \chi_{2}}(\Lambda)\right\| \geqq(1 / 4)$ so that $P_{\chi_{2} \chi_{2}}(\Lambda)$ is invertible, and

$$
\hat{G}_{\chi_{2} \chi_{2}}(\Lambda)=P_{\chi_{2} \chi_{2}}(\Lambda)\left[1+P_{\chi_{2} \chi_{2}}(\Lambda)^{-1} Q_{\chi_{2} \chi_{2}}(\Lambda)\right] .
$$

Using $\left|\hat{G}_{\chi_{2} \chi_{2}}\left(x_{0}, y_{0} ; \Lambda\right)\right| \leqq C_{\chi_{2} \chi_{2}} \prod_{x_{0} \leqq q<y_{0}}\left|w_{q} / \beta_{0}\right|^{6}$, we estimate $\left\|Q_{\chi_{2} \chi_{2}}(\Lambda)\right\|$ as follows:

$$
\left\|Q_{\chi_{2} \chi_{2}}(\Lambda)\right\| \leqq \max _{x_{0}} \sum_{y_{0}}\left|Q_{\chi_{2} \chi_{2}}\left(x_{0}, y_{0} ; \Lambda\right)\right| \leqq C_{\chi_{2} \chi_{2}} \max _{x_{0}} \sum_{y_{0} \neq x_{0}}\left(\beta_{1}^{\prime} / \beta_{0}\right)^{6\left|x_{0}-y_{0}\right|},
$$


assuming $\left|w_{q}\right|,|z|<\beta_{1}^{\prime}$. Thus

$$
\left\|Q_{\chi_{2} \chi_{2}}(\Lambda)\right\| \leqq 2 C_{\chi_{2} \chi_{2}} \frac{\left(\beta_{1}^{\prime} / \beta_{0}\right)^{6}}{1-\left(\beta_{1}^{\prime} / \beta_{0}\right)^{6}} .
$$

Choose $\beta_{1}^{\prime} \leqq \beta_{0}^{\prime}$ such that $\left\|Q_{\chi_{2} \chi_{2}}(\Lambda)\right\| \leqq 1 / 8$. Then $\left\|P_{\chi_{2} \chi_{2}}(\Lambda)^{-1} Q_{\chi_{2} \chi_{2}}(\Lambda)\right\| \leqq 1 / 2$, and $\hat{G}_{\chi_{2} \chi_{2}}(\Lambda)$ is invertible. Calling the inverse $-\hat{\Gamma}_{\chi_{2} \chi_{2}}(\Lambda)$, we have $\left\|\hat{\Gamma}_{\chi_{2} \chi_{2}}(\Lambda)\right\| \leqq 8$ if $\left|w_{q}\right|,|z|<\beta_{1}^{\prime}$. Since $\left.\hat{G}_{\chi_{2} \chi_{2}}\left(x_{0}, y_{0} ; \Lambda\right)\right|_{w_{q}=0}=0$ if $x_{0} \leqq q<y_{0}$, the same is true for $\left.\hat{\Gamma}_{\chi_{2} \chi_{2}}\left(x_{0}, y_{0} ; \Lambda\right)\right|_{w_{q}=0}$. From Leibniz's formula,

$$
\frac{\partial^{m}}{\partial w_{q}^{m}} \hat{\Gamma}_{\chi_{2} \chi_{2}}(\Lambda)=\sum_{n=0}^{m-1}\left(\begin{array}{c}
m \\
n
\end{array}\right) \frac{\partial^{n}}{\partial w_{q}^{n}} \hat{\Gamma}_{\chi_{2} \chi_{2}}(\Lambda) \frac{\partial^{m-n}}{\partial w_{q}^{m-n}} \hat{G}_{\chi_{2} \chi_{2}}(\Lambda) \hat{\Gamma}_{\chi_{2} \chi_{2}}(\Lambda)
$$

and Theorem 3.4, it follows immediately that

and

$$
\left.\frac{\partial^{m}}{\partial w_{q}^{m}} \hat{\Gamma}_{\chi_{2} \chi_{2}}(\Lambda)\right|_{w_{q}=0}=0 \quad \text { if } \quad 1 \leqq m \leqq 3,
$$

$$
\left.\frac{\partial^{m}}{\partial w_{q}^{m}} \hat{\Gamma}_{\chi_{2} \chi_{2}}(\Lambda)\right|_{w_{q}=0}=\left.\hat{\Gamma}_{\chi_{2} \chi_{2}}(\Lambda) \frac{\partial^{m}}{\partial w_{q}^{m}} \hat{G}_{\chi_{2} \chi_{2}}(\Lambda) \hat{\Gamma}_{\chi_{2} \chi_{2}}(\Lambda)\right|_{w_{q}=0} \quad(4 \leqq m \leqq 6) \text {. }
$$

Since $\left.\left(\partial^{m} / \partial w_{q}^{m}\right)\right|_{w_{q}=0} \hat{G}_{\chi_{2} \chi_{2}}\left(x_{0}, y_{0} ; \Lambda\right)=0$ if $x_{0} \leqq q<y_{0}$ and $m=4,5$, the same is true for $\left.\left(\partial^{m} / \partial w_{q}^{m}\right)\right|_{w_{q}=0} \hat{\Gamma}_{\chi_{2} \chi_{2}}\left(x_{0}, y_{0} ; \Lambda\right)$. In addition, if $x_{0} \leqq q<y_{0}$,

$$
\begin{aligned}
\frac{\partial^{6}}{\partial w_{q}^{6}} & \left.\hat{\Gamma}_{\chi_{2} \chi_{2}}\left(x_{0}, y_{0} ; \Lambda\right)\right|_{w_{q}=0} \\
= & \left.\sum_{\substack{u_{0} \leqq q \\
v_{0}>q}} \hat{\Gamma}_{\chi_{2} \chi_{2}}\left(x_{0}, u_{0} ; \Lambda\right) \frac{\partial^{6}}{\partial w_{q}^{6}} \hat{G}_{\chi_{2} \chi_{2}}\left(u_{0}, v_{0} ; \Lambda\right) \hat{\Gamma}_{\chi_{2} \chi_{2}}\left(v_{0}, y_{0} ; \Lambda\right)\right|_{w_{q}=0} \\
= & c_{7} \sum_{\substack{u_{0}, v_{0} \\
\Gamma_{\chi_{2} \chi_{2}}}}\left(x_{0}, u_{0} ; \Lambda\right) \hat{G}_{\chi_{2} \chi_{2}}\left(u_{0}, q ; \Lambda\right) \hat{G}_{\chi_{2} \chi_{2}}\left(q+1, v_{0} ; \Lambda\right) \\
& \left.\cdot \hat{\Gamma}_{\chi_{2} \chi_{2}}\left(v_{0}, y_{0} ; \Lambda\right)\right|_{w_{q}=0}=c_{7} \delta\left(x_{0}, q\right) \delta\left(q+1, y_{0}\right) .
\end{aligned}
$$

From the maximum modulus theorem,

$$
\left|\hat{\Gamma}_{\chi_{2} \chi_{2}}\left(x_{0}, y_{0} ; \Lambda\right)\right|=8 \prod_{x_{0} \leqq q<y_{0}}\left|w_{q} / \beta_{1}^{\prime}\right|^{7} \quad \text { if } \quad\left|x_{0}-y_{0}\right|>1,
$$

and letting all $w_{q}, z$ be equal to $\beta, 0<\beta<\beta_{1}^{\prime}$ :

$$
\left|\hat{\Gamma}_{\chi_{2} \chi_{2}}\left(x_{0}, y_{0} ; \Lambda\right)\right| \leqq 8\left(\beta / \beta_{1}^{\prime}\right)^{7\left|x_{0}-y_{0}\right|} \quad \text { if } \quad\left|x_{0}-y_{0}\right|>1 \text {. }
$$

It is not hard to show that $\lim _{\Lambda \rightarrow \mathbb{Z}^{3}} \hat{\Gamma}_{\chi_{2} \chi_{2}}\left(x_{0}, y_{0} ; \Lambda\right) \equiv \hat{\Gamma}_{\chi_{2} \chi_{2}}\left(y_{0}-x_{0}\right)$ exists and is the convolution inverse of $-\hat{G}_{\chi_{2} \chi_{2}}\left(x_{0}\right)$. We have $\left|\hat{\Gamma}_{\chi_{2} \chi_{2}}\left(x_{0}\right)\right| \leqq 8\left(\beta / \beta_{1}^{\prime}\right)^{7\left|x_{0}\right|}$ if $\left|x_{0}\right|>1$, so that $\hat{\Gamma}_{\chi_{2} \chi_{2}}\left(p_{0}\right)$ is analytic on $\left|\operatorname{Im} p_{0}\right| \leqq-7\left(1-\frac{\varepsilon}{2}\right) \log \beta$ if $\beta<\beta_{1}^{\prime 2 / \varepsilon} \equiv \beta_{3}$. The proof is complete.

Proof of Theorem 2.4. (a) We have $v_{2}\left(m_{2}\right) \sinh m_{2} /\left(\cosh m_{2}-1\right) \leqq \tilde{G}_{\chi_{2} \chi_{2}}\left(p_{0}=0\right)$ $\leqq C_{\chi_{2} \chi_{2}}$, so that $v_{2}\left(m_{2}\right) \leqq C_{\chi_{2} \chi_{2}} \operatorname{coth} m_{2}$. Choose $\beta_{2}^{\prime} \leqq \beta_{0}$ so small that $\operatorname{coth} m_{2}$ $<\sqrt{5 / 2}$ (for later convenience) for $0<\beta<\beta_{2}^{\prime}$. 
(b) From (2.14),

$$
\left|\tilde{H}_{2}\left(p_{0}\right)\right| \leqq \int_{-7\left(1-\frac{\varepsilon}{2}\right) \log \beta}^{\infty} \frac{\sinh \lambda_{0}}{\left|\cosh \lambda_{0}-\cos p_{0}\right|} d v_{2}\left(\lambda_{0}\right),
$$

and $\left|\operatorname{Im} p_{0}\right| \leqq-7(1-\varepsilon) \log \beta$. Choose $\beta_{3}^{\prime} \leqq \beta_{0}$ so small (depending on $\varepsilon$ ) that

$$
\cosh [-7(1-\varepsilon) \log \beta] \leqq \frac{1}{2} \cosh \left[-7\left(1-\frac{\varepsilon}{2}\right) \log \beta\right]
$$

for $0<\beta<\beta_{3}^{\prime}$. Then,

$$
\left|\cosh \lambda_{0}-\cos p_{0}\right| \geqq \cosh \lambda_{0}-\cosh \operatorname{Im} p_{0} \geqq \frac{1}{2} \cosh \lambda_{0}>\frac{1}{2}\left(\cosh \lambda_{0}-1\right)
$$

if $\quad \lambda_{0} \geqq-7\left(1-\frac{\varepsilon}{2}\right) \log \beta$. Therefore $\quad\left|\tilde{H}_{2}\left(p_{0}\right)\right| \leqq 2 \tilde{G}_{\chi_{2} \chi_{2}}\left(p_{0}=0\right) \leqq 2 C_{\chi_{2} \chi_{2}}$. Setting $\beta_{4}=\min \left\{\beta_{2}^{\prime}, \beta_{3}^{\prime}, \beta_{3}\right\}$ completes the proof of the theorem.

Proof of Theorem 2.5. Consider the finite volume approximation of $\hat{F}_{\varphi \varphi}^{(2)}\left(x_{0}\right)$. In matrix notation

$$
\hat{F}_{\varphi \varphi}^{(2)}(\Lambda)=\hat{G}_{\varphi \varphi}(\Lambda)+\hat{G}_{\varphi \chi_{2}}(\Lambda) \hat{\Gamma}_{\chi_{2} \chi_{2}}(\Lambda) \hat{G}_{\chi_{2} \varphi}(\Lambda)
$$

$\hat{F}_{\varphi \varphi}^{(2)}\left(x_{0}, y_{0} ; \Lambda\right)$ is analytic on $\left|w_{q}\right|,|z|<\beta_{1}^{\prime}$ (introduced in the proof of Theorem 2.3) and bounded there by $C_{\varphi \varphi}+8 C_{\varphi \chi_{2}} C_{\chi_{2} \varphi}$. Also, if all $w_{q}, z$ are equal to $\beta, 0<\beta<\beta_{1}^{\prime}$, $\lim _{\Lambda \rightarrow \mathbb{Z}^{3}} \hat{F}_{\varphi \varphi}^{(2)}\left(x_{0}, y_{0} ; \Lambda\right)=\hat{F}_{\varphi \varphi}^{(2)}\left(y_{0}-x_{0}\right)$. Clearly, if $x_{0} \leqq q<y_{0},\left.\hat{F}_{\varphi \varphi}^{(2)}\left(x_{0}, y_{0} ; \Lambda\right)\right|_{w_{q}=0}=0$. From

$$
\begin{aligned}
\frac{\partial^{m}}{\partial w_{q}^{m}} \hat{F}_{\varphi \varphi}^{(2)}(\Lambda)= & \frac{\partial^{m}}{\partial w_{q}^{m}} \hat{G}_{\varphi \varphi}(\Lambda) \\
& +\sum_{n_{1}+n_{2}+n_{3}=m} \frac{m !}{n_{1} ! n_{2} ! n_{3} !} \frac{\partial^{n_{1}}}{\partial w_{q}^{n_{1}}} \hat{G}_{\varphi \chi_{2}}(\Lambda) \frac{\partial^{n_{2}}}{\partial w_{q}^{n_{2}}} \hat{\Gamma}_{\chi_{2} \chi_{2}}(\Lambda) \frac{\partial^{n_{3}}}{\partial w_{q}^{n_{3}}} \hat{G}_{\chi_{2} \varphi}(\Lambda)
\end{aligned}
$$

and Theorem 3.4, we see that $\left.\left(\partial^{m} / \partial w_{q}^{m}\right) \hat{F}_{\varphi \varphi}^{(2)}(\Lambda)\right|_{w_{q}=0}=0$ if $1 \leqq m \leqq 3$, and that

$$
\begin{aligned}
\left.\frac{\partial^{m}}{\partial w_{q}^{m}} \hat{F}_{\varphi \varphi}^{(2)}(\Lambda)\right|_{w_{q}=0}= & \left.\frac{\partial^{m}}{\partial w_{q}^{m}} \hat{G}_{\varphi \varphi}(\Lambda)\right|_{w_{q}=0}+\left.\hat{G}_{\varphi \chi_{2}}(\Lambda) \hat{\Gamma}_{\chi_{2} \chi_{2}}(\Lambda) \frac{\partial^{m}}{\partial w_{q}^{m}} \hat{G}_{\chi_{2} \varphi}(\Lambda)\right|_{w_{q}=0} \\
& +\left.\hat{G}_{\varphi \chi_{2}}(\Lambda) \frac{\partial^{m}}{\partial w_{q}^{m}} \hat{\Gamma}_{\chi_{2} \chi_{2}}(\Lambda) \hat{G}_{\chi_{2} \varphi}(\Lambda)\right|_{w_{q}=0} \\
& +\left.\frac{\partial^{m}}{\partial w_{q}^{m}} \hat{G}_{\varphi \chi_{2}}(\Lambda) \hat{\Gamma}_{\chi_{2} \chi_{2}}(\Lambda) \hat{G}_{\chi_{2} \varphi}(\Lambda)\right|_{w_{q}=0}
\end{aligned}
$$

if $4 \leqq m \leqq 6$. Since $\varphi=P_{\mathbf{a}}^{(2)} \varphi$, Theorem 3.4 implies $\left.\left(\partial^{m} / \partial w_{q}^{m}\right)\right|_{w_{q}=0} \hat{G}_{\varphi \varphi}\left(x_{0}, y_{0} ; \Lambda\right)=0$, if $0 \leqq m \leqq 5$ and $x_{0} \leqq q<y_{0}$. Also $\left.\left(\partial^{2} / \partial w_{q}^{6}\right)\right|_{w_{q}=0} \hat{G}_{\varphi \varphi}\left(x_{0}, y_{0} ; \Lambda\right)=C_{7} \hat{G}_{\varphi \chi_{2}}\left(x_{0}, q ; \Lambda\right)$ - $\left.\hat{\hat{G}}_{\chi_{2} \varphi}\left(q+1, y_{0} ; \Lambda\right)\right|_{w_{q}=0}$, and a similar result holds for $\hat{G}_{\varphi \chi_{2}}, \hat{G}_{\chi_{2} \varphi}$. Hence, using the proof of Theorem 2.3 to calculate $\left.\left(\partial^{m} / \partial w_{q}^{m}\right)\right|_{w_{q}=0} \hat{\Gamma}_{\chi_{2} \chi_{2}}\left(x_{0}, y_{0} ; \Lambda\right)$, we find 
$\left.\left(\partial^{m} / \partial w_{q}^{m}\right)\right|_{w_{q}=0} \hat{F}_{\varphi \varphi}^{(2)}\left(x_{0}, y_{0} ; \Lambda\right)=0$ for $0 \leqq m \leqq 5$ and $x_{0} \leqq q<y_{0}$. In addition,

$$
\begin{aligned}
& \left.\frac{\partial^{6}}{\partial w_{q}^{6}} \hat{F}_{\varphi \varphi}^{(2)}\left(x_{0}, y_{0} ; \Lambda\right)\right|_{w_{q}=0}=\left.C_{7} \hat{G}_{\varphi \chi_{2}}\left(x_{0}, q ; \Lambda\right) \hat{G}_{\chi_{2} \varphi}\left(q+1, y_{0} ; \Lambda\right)\right|_{w_{q}=0} \\
& \quad+\left.\sum_{\substack{u_{0} \leqq q \\
v_{0} \leqq q}} \hat{G}_{\varphi \chi_{2}}\left(x_{0}, u_{0} ; \Lambda\right) \hat{\Gamma}_{\chi_{2} \chi_{2}}\left(u_{0}, v_{0} ; \Lambda\right) C_{7} \hat{G}_{\chi_{2} \chi_{2}}\left(v_{0}, q ; \Lambda\right) \hat{G}_{\chi_{2} \varphi}\left(q+1, y_{0} ; \Lambda\right)\right|_{w_{q}=0} \\
& \quad+\left.\sum_{\substack{u_{0} \leqq q \\
v_{0} \leqq q}} \hat{G}_{\varphi \chi_{2}}\left(x_{0}, u_{0} ; \Lambda\right) C_{7} \delta\left(u_{0}, q\right) \delta\left(q+1, v_{0}\right) \hat{G}_{\chi_{2} \varphi}\left(v_{0}, y_{0} ; \Lambda\right)\right|_{w_{q}=0} \\
& \quad+\left.\sum_{\substack{u_{0}>q \\
v_{0}>q}} C_{7} \hat{G}_{\varphi \chi_{2}}\left(x_{0}, q ; \Lambda\right) \hat{G}_{\chi_{2} \chi_{2}}\left(q+1, u_{0} ; \Lambda\right) \hat{\Gamma}_{\chi_{2} \chi_{2}}\left(u_{0}, v_{0} ; \Lambda\right) \hat{G}_{\chi_{2} \varphi}\left(v_{0}, y_{0} ; \Lambda\right)\right|_{w_{q}=0} \\
& =0 .
\end{aligned}
$$

Proceeding as in the proof of Theorem 2.1 , we conclude that $\tilde{F}_{\varphi \varphi}^{(2)}\left(p_{0}\right)$ is analytic up to $\left|\operatorname{Im} p_{0}\right|<-7 \log \left(\beta / \beta_{1}^{\prime}\right)$, which includes the region $\left|\operatorname{Im} p_{0}\right| \leqq-7\left(1-\frac{\varepsilon}{2}\right) \log \beta$ if
$\beta<\beta_{1}^{\prime 2 / \varepsilon} \equiv \beta_{3}$. The proof of the theorem is complete. $\square$

Proof of Theorem 2.7. Consider the finite volume approximation to $\hat{F}_{\chi_{1} \chi_{1}}\left(x_{0}\right)$ :

$$
\hat{F}_{\chi_{1} \chi_{1}}(\Lambda)=\hat{G}_{\chi_{1} \chi_{1}}(\Lambda)+\hat{G}_{\chi_{1} \chi}(\Lambda) \tilde{\Gamma}_{\chi \chi}(\Lambda) \hat{G}_{\chi_{\chi_{1}}}(\Lambda) \text {. }
$$

We assume $\left|w_{q}\right|,|z|<\beta_{5}$ (given in Theorem 2.6) which is so chosen that $\hat{\Gamma}_{\chi \chi}(\Lambda)$ is analytic there and $\left\|\hat{\Gamma}_{\chi \chi}(\Lambda)\right\| \leqq 4$ (see [5] or proceed as in the proof of Theorem 2.3). Thus, $\hat{F}_{\chi_{1} \chi_{1}}(\Lambda)$ is analytic on the region above and setting all $w_{q}, z$ equal to $\beta, 0<\beta$ $<\beta_{5}, \lim _{\Lambda \rightarrow \mathbb{Z}^{3}} \hat{F}_{\chi_{1} \chi_{1}}\left(x_{0}, y_{0}, \Lambda\right)=\hat{F}_{\chi_{1} \chi_{1}}\left(y_{0}-x_{0}\right)$. As in the proof of Theorem 2.3 , there exists $\hat{\beta}_{3}^{\prime} \leqq \beta_{5}$ such that $\hat{G}_{\chi_{1} \chi_{1}}(\Lambda)$ is invertible for $\left|w_{q}\right|,|z|<\beta_{3}^{\prime}$ and if $-\hat{\Gamma}_{\chi_{1} \chi_{1}}(\Lambda)$ denotes the inverse, $\left\|\hat{\Gamma}_{\chi_{1} \chi_{1}}(\Lambda)\right\| \leqq 8$. Hence, we can write

$$
\hat{F}_{\chi_{1} \chi_{1}}(\Lambda)=\hat{G}_{\chi_{1} \chi_{1}}(\Lambda)\left(1-\hat{\Gamma}_{\chi_{1} \chi_{1}}(\Lambda) \hat{G}_{\chi_{1} \chi}(\Lambda) \hat{\Gamma}_{\chi \chi}(\Lambda) \hat{G}_{\chi_{\chi_{1}}}(\Lambda)\right) .
$$

Now $\hat{G}_{\chi_{1} \chi}\left(\Lambda ;\left\{w_{q}=0\right\}, z=0\right)=0$, so that

$$
\hat{G}_{\chi_{1} \chi}(\Lambda)=\int_{0}^{1} d \lambda \frac{d}{d \lambda} \hat{G}_{\chi_{1} \chi}\left(\Lambda ;\left\{\lambda w_{q}\right\}, \lambda z\right) .
$$

The derivative can be estimated through a Cauchy integral as before. Thus, there exists $\beta_{4}^{\prime} \leqq \beta_{3}^{\prime}$ such that $\left\|\hat{G}_{\chi_{1} \chi}(\Lambda)\right\|,\left\|\hat{G}_{\chi_{\chi_{2}}}(\Lambda)\right\| \leqq 1 / 8$ for $\left|w_{q}\right|,|z|<\beta_{4}^{\prime}$. This implies $\hat{F}_{\chi_{1} \chi_{1}}(\Lambda)$ invertible. Calling the inverse $-\hat{\Phi}_{\chi_{1} \chi_{1}}(\Lambda)$, we have $\left\|\hat{\Phi}_{\chi_{1} \chi_{1}}(\Lambda)\right\| \leqq 16$. Setting all $w_{q}$, $z$ equal to $\beta, 0<\beta<\beta_{4}^{\prime}$, one can verify that $\lim _{\Lambda \rightarrow \mathbb{Z}^{3}} \hat{\Phi}_{\chi_{1} \chi_{1}}\left(x_{0}, y_{0} ; \Lambda\right) \equiv \hat{\Phi}_{\chi_{1} \chi_{1}}\left(y_{0}\right.$ $-x_{0}$ ) exists and is the convolution inverse of $-\hat{F}_{\chi_{1} \chi_{1}}\left(x_{0}\right)$. We now verify the analyticity properties of $\tilde{\Phi}_{\chi_{1} \chi_{1}}\left(p_{0}\right)$. From (4.6) and Theorem 3.4, it is clear that $\left.\hat{F}_{\chi_{1} \chi_{1}}\left(x_{0}, y_{0} ; \Lambda\right)\right|_{w_{q}=0}=0$, if $x_{0} \leqq q \leqq y_{0}$. Also $\left.\left(\partial^{m} / \partial w_{q}^{m}\right)\right|_{w_{q}=0} \hat{F}_{\chi_{1} \chi_{1}}(\Lambda)=0,1 \leqq m \leqq 3$ and

$$
\begin{aligned}
& \left.\frac{\partial^{m}}{\partial w_{q}^{m}} \hat{F}_{\chi_{1} \chi_{1}}(\Lambda)\right|_{w_{q}=0} \\
& =\left.\frac{\partial^{m}}{\partial w_{q}^{m}} \hat{G}_{\chi_{1} \chi_{1}}(\Lambda)\right|_{w_{q}=0}+\left.\hat{G}_{\chi_{1} \chi}(\Lambda) \hat{\Gamma}_{\chi \chi}(\Lambda) \frac{\partial^{m}}{\partial w_{q}^{m}} \hat{G}_{\chi \chi_{1}}(\Lambda)\right|_{w_{q}=0} \\
& \quad+\left.\hat{G}_{\chi_{1} \chi}(\Lambda) \frac{\partial^{m}}{\partial w_{q}^{m}} \hat{\Gamma}_{\chi \chi}(\Lambda) \hat{G}_{\chi \chi_{1}}(\Lambda)\right|_{w_{q}=0}+\left.\frac{\partial^{m}}{\partial w_{q}^{m}} \hat{G}_{\chi_{1} \chi}(\Lambda) \hat{\Gamma}_{\chi \chi}(\Lambda) \hat{G}_{\chi \chi_{1}}(\Lambda)\right|_{w_{q}=0}
\end{aligned}
$$


for $4 \leqq m \leqq 6$. To calculate these derivatives, we need $\left(\partial^{m} / \partial w_{q}^{m}\right) \hat{\Gamma}_{\chi \chi}$, which can be obtained as in the proof of Theorem 2.3:

$$
\left.\frac{\partial^{m}}{\partial w_{q}^{m}} \hat{\Gamma}_{\chi \chi}(\Lambda)\right|_{w_{q}=0}=\left.\hat{\Gamma}_{\chi \chi}(\Lambda) \frac{\partial^{m}}{\partial w_{q}^{m}} \hat{G}_{\chi \chi}(\Lambda) \hat{\Gamma}_{\chi \chi}(\Lambda)\right|_{w_{q}=0} \quad(4 \leqq m \leqq 6),
$$

hence, from Theorem 3.4, if $x_{0} \leqq q<y_{0}$,

$$
\left.\frac{\partial^{m}}{\partial w_{q}^{m}} \hat{\Gamma}_{\chi \chi}\left(x_{0}, y_{0} ; \Lambda\right)\right|_{w_{q}=0}=c_{m} \delta\left(x_{0}, q\right) \delta\left(q+1, y_{0}\right) ; \quad m=4,5
$$

and

$$
\begin{aligned}
\left.\frac{\partial^{6}}{\partial w_{q}^{\frac{6}{q}}} \hat{\Gamma}_{\chi \chi}\left(x_{0}, y_{0} ; \Lambda\right)\right|_{w_{q}=0}= & c_{6} \delta\left(x_{0}, q\right) \delta\left(q+1, y_{0}\right) \\
& +c_{7} \sum_{u_{0}, v_{0}} \hat{\Gamma}_{\chi \chi}\left(x_{0}, u_{0} ; \Lambda\right) \hat{G}_{\chi \chi_{1}}\left(u_{0}, q ; \Lambda\right) \\
& \left.\cdot \hat{G}_{\chi_{1} \chi}\left(q+1, v_{0} ; \Lambda\right) \hat{\Gamma}_{\chi \chi}\left(v_{0}, y_{0} ; \Lambda\right)\right|_{w_{q}=0} .
\end{aligned}
$$

Inserting (4.8), (4.9) into (4.7), we find if $x_{0} \leqq q<y_{0}$

$$
\left.\frac{\partial^{m}}{\partial w_{q}^{m}} \hat{F}_{\chi_{1} \chi_{1}}\left(x_{0}, y_{0} ; \Lambda\right)\right|_{w_{q}=0}=0 \quad(m=4,5)
$$

and after a lengthy computation

$$
\left.\frac{\partial^{6}}{\partial w_{q}^{6}} \hat{F}_{\chi_{1} \chi_{1}}\left(x_{0}, y_{0} ; \Lambda\right)\right|_{w_{q}=0}=\left.c_{7} \hat{F}_{\chi_{1} \chi_{1}}\left(x_{0}, q ; \Lambda\right) \hat{F}_{\chi_{1} \chi_{1}}\left(q+1, y_{0} ; \Lambda\right)\right|_{w_{q}=0} .
$$

As in the proof of Theorem 2.3 , these results imply $\left(x_{0} \leqq q<y_{0}\right)$

$$
\left.\frac{\partial^{m}}{\partial w_{q}^{m}} \hat{\Phi}_{\chi_{1} \chi_{1}}\left(x_{0}, y_{0} ; \Lambda\right)\right|_{w_{q}=0}=0 \quad \text { if } \quad 0 \leqq m \leqq 5
$$

and

$$
\left.\frac{\partial^{6}}{\partial w_{q}^{6}} \hat{\Phi}_{\chi_{1} \chi_{1}}\left(x_{0}, y_{0} ; \Lambda\right)\right|_{w_{q}=0}=c_{7} \delta\left(x_{0}, q\right) \delta\left(q+1, y_{0}\right) .
$$

By the same arguments as before, $\tilde{\Phi}_{\chi_{1} \chi_{1}}\left(p_{0}\right)$ is thus analytic on $\left|\operatorname{Im} p_{0}\right|$ $<-7 \log \left(\beta / \beta_{4}^{\prime}\right)$, which includes $\left|\operatorname{Im} p_{0}\right|<-7\left(1-\frac{\varepsilon}{2}\right) \log \beta$ if $\beta<\beta_{4}^{\prime 2 / \varepsilon}$. Setting $\beta_{6}$ $=\beta_{4}^{\prime 2 / \varepsilon}$ completes the proof of the theorem.

Proof of Theorem 2.8. Consider the finite volume approximation to $\hat{K}\left(x_{0}\right)$ :

$$
\hat{K}(\Lambda)=\hat{\Gamma}_{\chi_{2} \chi_{2}}(\Lambda)-\hat{\Phi}_{\chi_{2} \chi_{2}}(\Lambda)
$$

We assume $\left|w_{q}\right|,|z|<\beta_{6}$ so that the bound $\|\hat{K}(\Lambda)\| \leqq 24$ holds. Also, from the proof of Theorems 2.3 and 2.7, if $x_{0} \leqq q<y_{0}$,

$$
\left.\left(\partial^{m} / \partial w_{q}^{m}\right)\right|_{w_{q}=0} \hat{K}\left(x_{0}, y_{0} ; \Lambda\right)=0, \quad 0 \leqq m \leqq 5 .
$$


But also, due to (4.5) and (4.10)

$$
\left.\frac{\partial^{6}}{\partial w_{q}^{6}} \hat{K}\left(x_{0}, y_{0} ; \Lambda\right)\right|_{w_{q}=0}=0 \quad \text { if } \quad x_{0} \leqq q<y_{0} .
$$

This implies in the usual way $\left|\hat{K}\left(x_{0}\right)\right| \leqq 24\left(\beta / \beta_{6}\right)^{7\left|x_{0}\right|}$, and $\tilde{K}\left(p_{0}\right)$ is analytic on $\left|\operatorname{Im} p_{0}\right|$ $<-7\left(1-\frac{\varepsilon}{2}\right) \log \beta$ provided $\beta<\beta_{6}^{2 / \varepsilon}$. In addition, $\hat{K}\left(\Lambda ;\left\{w_{q}=0\right\}, z=0\right)=0$ and by doing an estimate using the Cauchy formula as before, we find $\|\hat{K}(\Lambda)\|$ $\leqq 96 \max \left\{\left|w_{q}\right| / \beta_{6},|z| / \beta_{6}\right\}$, if $\left|w_{q}\right|,|z|<\beta_{6} / 2$. This is automatically satisfied if $\left|w_{q}\right|,|z|$ $<\beta_{6}^{2 / \varepsilon}$, since we assumed from the beginning that $\beta_{0} \leqq 1 / 2$ and $\varepsilon \leqq 1 / 10$. In particular, $\left|\hat{K}\left(x_{0}=0\right)\right| \leqq 96\left(\beta / \beta_{6}\right)$ for $0<\beta<\beta_{6}^{2 / \varepsilon}$. Hence, if $\left|\operatorname{Im} p_{0}\right| \leqq-7(1-\varepsilon) \log \beta$,

$$
\begin{aligned}
\left|\tilde{K}\left(p_{0}\right)\right| & \leqq 96\left[\left(\beta / \beta_{6}\right)+2 \sum_{n=1}^{\infty} \exp \left(7 \log \frac{\beta}{\beta_{6}}+\left|\operatorname{Im} p_{0}\right|\right) n\right] \\
& \leqq 96\left[\left(\beta / \beta_{6}\right)+2 \sum_{n=1}^{\infty} \exp \left(7\left(1-\frac{\varepsilon}{2}\right) \log \beta-7(1-\varepsilon) \log \beta\right) n\right] \\
& =96\left[\frac{\beta}{\beta_{6}}+\frac{2 \exp \left(\frac{7}{2} \varepsilon \log \beta\right)}{1-\exp \left(\frac{7}{2} \varepsilon \log \beta\right)}\right] \leqq 96\left[\frac{\beta}{\beta_{6}}+4 \exp \left(\frac{7}{2} \varepsilon \log \beta\right)\right],
\end{aligned}
$$

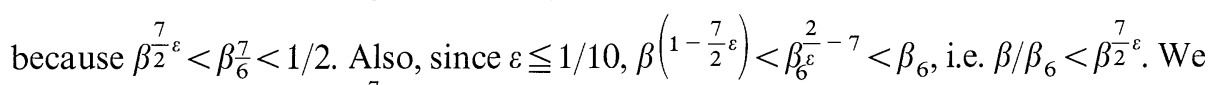
thus have $\left|\tilde{K}\left(p_{0}\right)\right| \leqq 480 \beta^{\frac{7}{2} \varepsilon}$ and therefore, from Theorem $2.4\left|\tilde{T}_{2}\left(p_{0}\right)\right| \leqq 480 k_{4} \beta^{(7 \varepsilon / 2)}$ $\leqq 1 / 2$ if $\beta \leqq \beta_{5}^{\prime}$ (this defines $\beta_{5}^{\prime}$ ). Letting $\beta_{7}=\min \left\{\beta_{6}^{2 / \varepsilon}, \beta_{5}^{\prime}\right\}$ completes the proof of the theorem.

Proof of Theorem 2.9. From Theorems 2.4 and 2.8, $\left.\mid v_{2} \underset{\sim}{\left(m_{2}\right.}\right)\left(1+\tilde{T}_{2}\right)^{-1} \tilde{K} \mid \leqq 2 k_{3} k_{5} \beta^{\frac{7}{2}}{ }^{\varepsilon}$ on $\left|\operatorname{Im} p_{0}\right| \leqq-7(1-\varepsilon) \log \beta$, if $\beta<\beta_{7}$. Thus, $\left|v_{2}\left(m_{2}\right)\left(1+\tilde{T}_{2}\right)^{-1} \tilde{K}\right| \leqq(1 / 2)$ if $\beta<\beta_{6}^{\prime} \leqq \beta_{7}$ for an appropriate $\beta_{6}^{\prime}$. Let

and

$$
\begin{gathered}
A B=\left\{p_{0}:\left|\operatorname{Re} p_{0}\right| \leqq \pi, \operatorname{Im} p_{0}=0\right\} ; \\
B C=\left\{p_{0}: \operatorname{Re} p_{0}=\pi, 0 \leqq \operatorname{Im} p_{0} \leqq-7(1-\varepsilon) \log \beta\right\} ; \\
C D=\left\{p_{0}:\left|\operatorname{Re} p_{0}\right| \leqq \pi, \operatorname{Im} p_{0}=-7(1-\varepsilon) \log \beta\right\}
\end{gathered}
$$

On $A B$,

$$
A D=\left\{p_{0}: \operatorname{Re} p_{0}=-\pi, 0 \leqq \operatorname{Im} p_{0} \leqq-7(1-\varepsilon) \log \beta\right\}
$$

$$
\left|g\left(p_{0}\right)\right|=\left(\cosh m_{2}-\cos p_{0}\right) / \sinh m_{2} \geqq 1-\left(1 / \sinh m_{2}\right)>1 / 2,
$$

since from the proof of Theorem $2.4 \operatorname{coth} m_{2}<\sqrt{5} / 2$ which implies $1 / \sinh m_{2}<1 / 2$.

On $B C, \quad\left|g\left(p_{0}\right)\right|=\left(\cosh m_{2}+\cosh \operatorname{Im} p_{0}\right) / \sinh m_{2} \geqq 1$, and similarly on $A D$. Finally, on $C D$,

$$
\begin{aligned}
\left|g\left(p_{0}\right)\right|^{2}= & {\left[\cosh ^{2} m_{2}+\cos ^{2} \operatorname{Re} p_{0}+\sinh ^{2} 7(1-\varepsilon) \log \beta\right.} \\
& \left.-2 \cosh m_{2} \cos \operatorname{Re} p_{0} \cosh 7(1-\varepsilon) \log \beta\right] / \sinh ^{2} m_{2} \\
\geqq & {\left[\left(\cosh m_{2}-\cosh 7(1-\varepsilon) \log \beta\right)^{2}-1\right] / \sinh ^{2} m_{2} } \\
\geqq & \left(\frac{\cosh 7(1-\varepsilon) \log \beta}{\sinh m_{2}}-\operatorname{coth} m_{2}\right)^{2}-\frac{1}{4}
\end{aligned}
$$


The right-hand side tends to infinity as $\beta \rightarrow 0$. Thus, it stays bigger than $1 / 2$ if $\beta<\beta_{7}^{\prime}$ $\leqq \beta_{6}^{\prime}$ for an appropriate $\beta_{7}^{\prime}$. We set $\beta_{8} \equiv \beta_{7}^{\prime}$ to finish the proof.

Proof of Theorem 2.11. Let $p_{0}=i m_{2}+r$, with $|r|=\zeta \beta$. $\zeta$ will be given explicitly below. Then,

$$
\left|g\left(p_{0}\right)\right|=|r| \operatorname{coth} m_{2}(1-\cos r) / r+i \sin r / r \mid
$$

and $\lim _{\beta \rightarrow 0}\left|g\left(p_{0}\right)\right| /|r|=1$. We choose $\beta_{8}^{\prime} \leqq \beta_{8}$ such that $|r| \leqq 1$ and $\left|g\left(p_{0}\right)\right|>(1 / 2) r$. On the other hand, from the proof of Theorem 2.8,

$$
\left|\tilde{K}\left(p_{0}\right)\right| \leqq 96\left[\left(\beta / \beta_{6}\right)+2 \sum_{n=1}^{\infty} \exp \left[\left(7 \log \left(\beta / \beta_{6}\right)+m_{2}+1\right) n\right]\right] .
$$

From Theorem 2.2, $m_{2} \leqq-6 \log \left(d_{1} \beta\right)$, hence

$$
\begin{aligned}
\left|\tilde{K}\left(p_{0}\right)\right| & \leqq 96\left[\left(\beta / \beta_{6}\right)+2 \sum_{n=1}^{\infty} \exp \left[\left(\log \beta-\log \left(\beta_{6}^{7} d_{1}^{6}\right)+1\right) n\right]\right] \\
& =96\left[\left(\beta / \beta_{6}\right)+2 \frac{\left(e \beta / \beta_{6}^{7} d_{1}^{6}\right)}{1-\left(e \beta / \beta_{6}^{7} d_{1}^{6}\right)}\right] \\
& \leqq 96\left[\left(\beta / \beta_{6}\right)+4 e \beta /\left(\beta_{6}^{7} d_{1}^{6}\right)\right]
\end{aligned}
$$

if $\beta<\beta_{9} \leqq \beta_{8}^{\prime}$ is appropriately chosen. We set $\zeta=192\left[1 / \beta_{6}+4 e /\left(\beta_{6}^{7} d_{1}^{6}\right)\right]$, so that $\left|\tilde{K}\left(p_{0}\right)\right| \leqq(1 / 2) \zeta \beta$. In conclusion, we have $\left|\tilde{K}\left(p_{0}\right)\right| \leqq(1 / 2) \zeta \beta=(1 / 2)|r|<\left|g\left(p_{0}\right)\right|$ and the proof is complete.

Proof of Theorem 2.13. Consider the finite volume approximations to $\hat{L}_{\chi_{1} \chi}\left(x_{0}\right)$ :

$$
\hat{L}_{\chi_{1} \chi}(\Lambda)=\hat{\Phi}_{\chi_{1} \chi_{1}}(\Lambda) \hat{G}_{\chi_{1} \chi}(\Lambda) \hat{\Gamma}_{\chi \chi}(\Lambda),
$$

where $\left|w_{q}\right|,|z|<\beta_{6}$, so that $\left\|\hat{\Phi}_{\chi_{1} \chi_{1}}(\Lambda)\right\| \leqq 16,\left\|\hat{G}_{\chi_{1} \chi}(\Lambda)\right\| \leqq C_{\chi_{1} \chi}$, and $\left\|\hat{\Gamma}_{\chi \chi}(\Lambda)\right\| \leqq 4$, hence $\left\|\hat{L}_{\chi_{1} \chi}^{q}(\Lambda)\right\| \leqq 64 C_{\chi_{1} \chi}$. From (4.12) it follows that $\left.\hat{L}_{\chi_{1} \chi}\left(x_{0}, y_{0} ; \Lambda\right)\right|_{w_{q}=0}=0$, if $x_{0}$ $\leqq q<y_{0}$ and $\left.\left(\partial^{m} / \partial w_{q}^{m}\right)\right|_{w_{q}=0} \hat{L}_{\chi_{1} \chi}(\Lambda)=0$, if $1 \leqq m \leqq 3$. Also,

$$
\begin{aligned}
\left.\frac{\partial^{m}}{\partial w_{q}^{m}} \hat{L}_{\chi_{1} \chi}(\Lambda)\right|_{w_{q}=0}= & \left.\hat{\Phi}_{\chi_{1} \chi_{1}}(\Lambda) \hat{G}_{\chi_{1} \chi}(\Lambda) \frac{\partial^{m}}{\partial w_{q}^{m}} \hat{\Gamma}_{\chi \chi}(\Lambda)\right|_{w_{q}=0} \\
& +\left.\hat{\Phi}_{\chi_{1} \chi_{1}}(\Lambda) \frac{\partial^{m}}{\partial w_{q}^{m}} \hat{G}_{\chi_{1} \chi}(\Lambda) \hat{\Gamma}_{\chi \chi}(\Lambda)\right|_{w_{q}=0} \\
& +\left.\frac{\partial^{m}}{\partial w_{q}^{m}} \hat{\Phi}_{\chi_{1} \chi_{1}}(\Lambda) \hat{G}_{\chi_{1} \chi}(\Lambda) \hat{\Gamma}_{\chi \chi}(\Lambda)\right|_{w_{q}=0} . \quad(4 \leqq m \leqq 6) .
\end{aligned}
$$

A straightforward but tedius computation shows that

$$
\left.\frac{\partial^{m}}{\partial w_{q}^{m}} \hat{L}_{\chi_{1} \chi}\left(x_{0}, y_{0} ; \Lambda\right)\right|_{w_{q}=0}=0, \quad \text { if } \quad x_{0} \leqq q<y_{0} \quad \text { and } \quad 4 \leqq m \leqq 6 .
$$

Thus, in the usual way this implies $\left|\hat{L}_{\chi_{1} x}\left(x_{0}\right)\right| \leqq 64 C_{\chi_{1} \chi}\left(\beta / \beta_{6}\right)^{7\left|x_{0}\right|}$ if $0<\beta<\beta_{6}$ and $\tilde{L}_{\chi_{1} \chi}\left(p_{0}\right)$ analytic up to $\left|\operatorname{Im} p_{0}\right|<-7(1-\varepsilon / 2) \log \beta$ if $0<\beta<\beta_{7}$ (remember $\left.\beta_{7} \leqq \beta_{6}^{2 / \varepsilon}\right)$. Also, $\hat{L}_{\chi_{1} \chi}\left(\Lambda ;\left\{w_{q}=0\right\}, z=0\right)=0$. Proceeding in the same way as for $\hat{K}(\Lambda)$, we deduce, after an estimate using the Cauchy formula, that $\left|\hat{L}_{\chi_{1} \chi}\left(x_{0}=0\right)\right|$ 
$\leqq 256 C_{\chi_{1 x}}\left(\beta / \beta_{6}\right)$ if $0<\beta<\beta_{7}$, and this leads again as in the proof of Theorem 2.8 to $\left|\tilde{L}_{\chi_{1} \chi}\left(p_{0}\right)\right| \leqq 1280 C_{\chi_{1} \alpha} \beta^{\frac{7}{2} \varepsilon}$ for $\left|\operatorname{Im} p_{0}\right| \leqq-7(1-\varepsilon) \log \beta$.

Proof of Theorem 2.14. Let

$$
\hat{M}(\Lambda)=\hat{\Gamma}_{\chi \chi}(\Lambda)-\hat{L}_{\chi \chi_{1}}(\Lambda) \hat{F}_{\chi_{1} \chi_{1}}(\Lambda) \hat{L}_{\chi_{1} \chi}(\Lambda)
$$

with $\left|w_{q}\right|,|z|<\beta_{6}$. Then $\left\|\hat{\Gamma}_{\chi \chi}(\Lambda)\right\| \leqq 4,\left\|\hat{L}_{\chi \chi_{1}}(\Lambda)\right\| \leqq 64 C_{\chi \chi_{1}}$ and $\left\|\hat{F}_{\chi_{1} \chi_{1}}(\Lambda)\right\| \leqq C_{\chi_{1} \chi_{1}}$ $+4 C_{\chi_{1} \chi} C_{\chi \chi_{1}}$ [see (4.6)], so that $\|\hat{M}(\Lambda)\| \leqq k_{M}$ for and appropriate constant. Now, if $x_{0} \leqq q<y_{0}$, one shows by direct calculation that

$$
\left.\frac{\partial^{m}}{\partial w_{q}^{m}} \hat{M}\left(x_{0}, y_{0} ; \Lambda\right)\right|_{w_{q}=0}=0 \quad \text { for } \quad 0 \leqq m \leqq 3,
$$

and

$$
\left.\frac{\partial^{m}}{\partial w_{q}^{m}} \hat{M}\left(x_{0}, y_{0} ; \Lambda\right)\right|_{w_{q}=0}=c_{m} \delta\left(x_{0}, q\right) \delta\left(q+1, y_{0}\right) \quad \text { for } \quad 4 \leqq m \leqq 6 .
$$

This implies, as before, that $\left|\hat{M}\left(x_{0}\right)\right| \leqq k_{M}\left(\beta / \beta_{6}\right)^{7\left|x_{0}\right|}$ if $\left|x_{0}\right|>1$ if $0<\beta<\beta_{6}$, and therefore $\tilde{M}\left(p_{0}\right)$ is analytic on

$$
\left|\operatorname{Im} p_{0}\right|<-7(1-\varepsilon / 2) \log \beta \quad \text { if } \quad 0<\beta<\beta_{7} .
$$

Proof of Theorem 2.15. Consider

$$
\hat{G}_{x \chi_{1}}\left(x_{0}, x_{0} ; \Lambda:\left\{w_{q}=0\right\}, z\right)=\hat{G}_{\chi x_{h}}\left(x_{0}, x_{0} ; \Lambda ;\left\{w_{q}=0\right\}, z\right) .
$$

It is clear that

$$
\begin{aligned}
& \lim _{z \rightarrow 0} \hat{G}_{\chi \chi_{1}}\left(x_{0}, x_{0} ; \Lambda ;\left\{w_{q}=0\right\}, z\right) / z \\
& \quad=2 \int \chi\left(g_{\mathscr{P}_{\ell}}\right) \chi_{h}(g) \chi\left(g_{P_{r}}\right) d g_{\Lambda}=(2 / d)
\end{aligned}
$$

( $d$ =dimension of the representation $\chi$ ). Here, $P_{\ell}$ and $P_{r}$ are the left and right plaquettes of the elementary horizontal window. From (4.12),

$$
\begin{aligned}
\hat{L}_{\chi \chi_{1}} & \left(x_{0}, x_{0} ; \Lambda ;\left\{w_{q}=0\right\}, z\right) \\
= & \hat{\Gamma}_{\chi \chi}\left(x_{0}, x_{0} ; \Lambda ;\left\{w_{q}=0\right\}, z\right) \\
& \quad \hat{G}_{\chi \chi_{1}}\left(x_{0}, x_{0} ; \Lambda ;\left\{w_{q}=0\right\}, z\right) \hat{\Phi}_{\chi_{1} \chi_{1}}\left(x_{0}, x_{0} ; \Lambda ;\left\{w_{q}=0\right\}, z\right),
\end{aligned}
$$

and hence

$$
\begin{aligned}
& \lim _{z \rightarrow 0} z^{-1} \hat{L}_{\chi \chi_{1}}\left(x_{0}, x_{0} ; \Lambda ;\left\{w_{q}=0\right\}, z\right) \\
& \quad=2 \lim _{z \rightarrow 0} z^{-1} \hat{G}_{\chi \chi_{1}}\left(x_{0}, x_{0} ; \Lambda ;\left\{w_{q}=0\right\}, z\right)=(4 / d) .
\end{aligned}
$$

Using the fact that $\left.\left(\partial / \partial w_{q}\right)\right|_{w_{q}=0} \hat{L}_{x \chi_{1}}(\Lambda)=0$ for all $q$, the above result implies

$$
\left.\frac{d}{d \beta} \hat{L}_{x x_{1}}\left(x_{0}, x_{0} ; \Lambda ;\left\{w_{q}=\beta\right\}, z=\beta\right)\right|_{\beta=0}=\frac{4}{d},
$$


from which follows (taking the thermodynamic limit)

$$
\left.\frac{d}{d \beta} \hat{L}_{\chi \chi_{1}}\left(x_{0}=0\right)\right|_{\beta=0}=\frac{4}{d} .
$$

We choose $\beta<\beta_{9}^{\prime}\left(\leqq \beta_{9}\right)$ so that $\left|\hat{L}_{x x_{1}}\left(x_{0}=0\right)\right| \geqq(2 / d) \beta$.

Now, consider $\hat{L}_{\chi \chi_{1}}\left(x_{0}=0, y_{0}=1 ; \Lambda ;\left\{w_{q}=0\right\}_{q \neq 0}, z=0\right)$. We claim that this is identically zero as a function of $w_{0}$. The reason is that $\hat{G}_{\chi x_{1}}\left(x_{0}, y_{0} ; \Lambda ;\left\{w_{q}=0\right\}_{q \neq 0}\right.$, $z=0)=0$ for all $x_{0}, y_{0}$. This is clear if $x_{0}<0$ or $y_{0}>1$ (or vice-versa), but is also true when $x_{0}=0$ and $y_{0}=1$ because integrals of the form

$$
\int \chi(0, \mathbf{0}) \chi_{h}(1, \mathbf{x}) \exp \left[w_{0} \sum_{P \in \mathscr{P}_{0}^{\prime \prime}} \chi\left(g_{P}\right)\right] d g_{\Lambda}
$$

vanish identically as is easy to see.

Write

$$
\hat{L}_{\chi \chi_{1}}\left(x_{0}=0, y_{0}=1 ; \Lambda ;\left\{w_{q}\right\}, z\right)=\sum_{n=7}^{\infty} h_{n}\left(\Lambda ;\left\{w_{q}\right\}_{q \neq 0}, z\right) w_{0}^{n},
$$

with

$$
h_{n}\left(\Lambda ;\left\{w_{q}\right\}_{q \neq 0}, z\right)=\left.\frac{1}{h !} \frac{\partial^{n}}{\partial w_{0}^{n}} \hat{L}_{\chi \chi_{1}}\left(x_{0}=0, y_{0}=1 ; \Lambda ;\left\{w_{q}\right\}, z\right)\right|_{w_{q}=0} .
$$

From the remark above, $h_{n}\left(\Lambda ;\left\{w_{q}=0\right\}_{q \neq 0}, z=0\right)=0 \forall n$. Setting all $w_{q}$, $z$ equal to the same $\beta$ in (4.14), we get

$$
\hat{L}_{\chi \chi_{1}}\left(x_{0}=0, y_{0}=1 ; \Lambda ; \beta\right)=\sum_{n=7}^{\infty} h_{n}(\Lambda ; \beta) \beta^{n},
$$

and $h_{n}(\Lambda ; \beta=0)=0$. From this, we get the important result

$$
\left.\frac{\partial^{7}}{\partial \beta^{7}} \hat{L}_{x x_{1}}\left(x_{0}=0, y_{0}=1 ; \Lambda ; \beta\right)\right|_{\beta=0}=0,
$$

which implies, if $|\beta|<\beta_{6}:\left|\hat{L}_{x \chi_{1}}\left(x_{0}=0, y_{0}=1 ; \Lambda\right)\right| \leqq 64 C_{x x_{1}}\left|\beta / \beta_{6}\right|^{8}$. This bound carries to the infinite volume limit:

$$
\left|\hat{L}_{\chi \chi_{1}}\left(x_{0}=1\right)\right| \leqq 64 C_{\chi \chi_{1}}\left(\beta / \beta_{6}\right)^{8} \quad \text { if } \quad 0<\beta<\beta_{6} .
$$

Now, suppose $p_{0}=i m_{2}+r$, with $|r| \leqq 1$. Then,

$$
\begin{aligned}
& \left|\tilde{L}_{x x_{1}}\left(p_{0}\right)-\hat{L}_{x \chi_{1}}\left(x_{0}=0\right)\right| \\
& \quad \leqq\left|\sum_{\left|x_{0}\right|=1} \hat{L}_{x x_{1}}\left(x_{0}\right) e^{i p_{0} x_{0}}+\sum_{\left|x_{0}\right| \geqq 2} \hat{L}_{x x_{1}}\left(x_{0}\right) e^{i p_{0} x_{0}}\right| \\
& \quad \leqq 128 C_{\chi \chi_{1}}\left(\frac{\beta}{\beta_{6}}\right)^{8} \exp \left(m_{2}+1\right)+128 C_{\chi x_{1}} \sum_{n=2}^{\infty} \exp \left[\left(7 \log \left(\frac{\beta}{\beta_{6}}\right)+m_{2}+1\right) n\right],
\end{aligned}
$$


where we used results from the proof of Theorem 2.13. Proceeding now as in the proof of Theorem 2.11, using $m_{2} \leqq-6 \log \left(d_{1} \beta\right)$, we get

$$
\begin{aligned}
& \left|\tilde{L}_{\chi \chi_{1}}\left(p_{0}\right)-\hat{L}_{\chi \chi_{1}}\left(x_{0}=0\right)\right| \\
& \quad \leqq 128 C_{\chi \chi_{1}}\left[\beta^{2}\left(\frac{e}{\beta_{6}^{8} d_{1}^{6}}\right)+\frac{\left(e \beta / \beta_{6}^{7} d_{1}^{6}\right)^{2}}{1-\left(e \beta / \beta_{6}^{7} d_{1}^{6}\right)}\right] \\
& \quad \leqq 128 C_{\chi \chi_{1}}\left[\left(\frac{e}{\left.\beta_{6}^{8} d_{1}^{6}\right)}\right)+2\left(\frac{e}{\left.\beta_{6}^{7} d_{1}^{6}\right)}\right)^{2}\right] \beta^{2} \equiv k_{L} \beta^{2} \text { if } \beta<\beta_{9} .
\end{aligned}
$$

Thus, if $\beta<\beta_{9}^{\prime}$, we have

$$
\left|\tilde{L}_{\chi \chi 1}\left(p_{0}\right)\right| \geqq(2 / d) \beta-k_{L} \beta^{2},
$$

and we choose $\beta_{10} \leqq \beta_{9}^{\prime}$ so that $\left|\tilde{L}_{\chi \chi_{1}}\left(p_{0}\right)\right|>0$ for $0<\beta<\beta_{10}$.

Proof of Theorem 2.16. First, notice that if $0<\beta<\beta_{7},\left|\tilde{L}_{\chi \chi_{1}} \tilde{H}_{2}\left(1+\tilde{T}_{2}\right)^{-1} \tilde{L}_{\chi_{1} \chi}\right| \leqq a \beta^{7 \varepsilon}$ for some constant $a$ and $\left|\operatorname{Im} p_{0}\right| \leqq-7(1-\varepsilon) \log \beta$. This follows from Theorems 2.4, 2.8, and 2.13. From the proof of Theorem 2.14, $\left|\hat{M}\left(x_{0}\right)\right| \leqq k_{M}\left(\beta / \beta_{6}\right)^{7\left|x_{0}\right|}$ if $\left|x_{0}\right|>1$. Thus,

$$
\tilde{M}\left(p_{0}\right)=\hat{M}\left(x_{0}=0\right)+\sum_{\left|x_{0}\right|=1} \hat{M}\left(x_{0}\right) e^{i p_{0} x_{0}}+\tilde{M}_{1}\left(p_{0}\right)
$$

with $\tilde{M}_{1}\left(p_{0}\right)=\sum_{\left|x_{0}\right| \geqq 2} \hat{M}\left(x_{0}\right) e^{i p_{0} x_{0}}$ being bounded on $\left|\operatorname{Im} p_{0}\right| \leqq-7(1-\varepsilon) \log \beta$ by

$$
\left|\hat{M}_{1}\left(p_{0}\right)\right| \leqq \sum_{\left|x_{0}\right| \geqq 2} k_{M} \exp \left[\left(7 \log \left(\beta / \beta_{6}\right)-7(1-\varepsilon) \log \beta\right)\left|x_{0}\right|\right] \leqq 4 k_{M} \beta^{7 \varepsilon}
$$

(as in the proof of Theorem 2.8). Now, from the definition (4.13),

$$
\hat{M}\left(\Lambda ;\left\{w_{q}=0\right\}, z=0\right)=\hat{\Gamma}_{\chi \chi}\left(\Lambda ;\left\{w_{q}=0\right\}, z=0\right)=-I,
$$

so that $\lim _{\beta \rightarrow 0} \hat{M}\left(x_{0}=0\right)=-1$. Also, from the results in the proof of Theorem 2.15,

$$
\begin{aligned}
\hat{M}\left(x_{0}=0, y_{0}=1 ; \Lambda ;\left\{w_{q}=0\right\}_{q \neq 0}, z=0\right) & \hat{\Gamma}_{\chi \chi}\left(x_{0}=0, y_{0}=1 ; \Lambda ;\left\{w_{q}=0\right\}_{q \neq 0}, z=0\right) \\
& -\hat{L}_{\chi \chi_{1}}\left(x_{0}=0, y_{0}=0 ; \Lambda ;\left\{w_{q}=0\right\}_{q \neq 0}, z=0\right) \\
& \cdot \hat{F}_{\chi_{1 \chi_{1}}}\left(x_{0}=0, y_{0}=1 ; \Lambda ;\left\{w_{q}=0\right\}_{q \neq 0}, z=0\right) \\
& \cdot \hat{L}_{\chi_{1 \chi}}\left(x_{0}=1, y_{0}=1 ; \Lambda ;\left\{w_{q}=0\right\}_{q \neq 0}, z=0\right) .
\end{aligned}
$$

From the proof of Theorem 2.7, we know that

$$
\hat{F}_{\chi_{1} \chi_{1}}\left(x_{0}=0, y_{0}=1 ; \Lambda ;\left\{w_{q}=\right\}_{q \neq 0}, z=0\right) \text { is } O\left(w_{0}^{6}\right) \text {. }
$$

Also, from

$$
\left.\frac{\partial^{4}}{\partial w_{0}^{4}} \hat{\Gamma}_{\chi \chi}(\Lambda)\right|_{w_{q}=0}=\left.\hat{\Gamma}_{\chi \chi}(\Lambda) \frac{\partial^{4}}{\partial w_{0}^{4}} \hat{G}_{\chi \chi}(\Lambda) \hat{\Gamma}_{\chi \chi}(\Lambda)\right|_{w_{q}=0},
$$

we have

$$
\left.\left(\partial^{4} / \partial w_{0}^{4}\right)\right|_{w_{0}=0} \hat{\Gamma}_{\chi \chi}\left(x_{0}=0, y_{0}=1 ; \Lambda ;\left\{w_{q}=0\right\}_{q \neq 0}, z=0\right)=c_{4}
$$


i.e.

$$
\hat{\Gamma}_{\chi \chi}\left(x_{0}=0, y_{0}=1 ; \Lambda ;\left\{w_{q}=0\right\}_{q \neq 0}, z=0\right)=O\left(w_{0}^{4}\right) .
$$

Therefore,

$$
\lim _{w_{0} \rightarrow 0} w_{0}^{-4} \tilde{M}\left(x_{0}=0, y_{0}=1 ; \Lambda ;\left\{w_{q}=0\right\}_{q \neq 0}, z=0\right)=c_{4} / 4 !,
$$

which implies

$$
\lim _{\beta \rightarrow 0} \beta^{-4} \tilde{M}\left(x_{0}=0, y_{0}=1 ; \Lambda ; \beta\right)=c_{4} / 4 !
$$

In this last expression, $\hat{M}$ is calculated setting all $w_{q}, z$ equal to the same $\beta$. Taking the thermodynamic limit, we conclude that

$$
\lim _{\beta \rightarrow 0} \frac{1}{\beta^{4}} \hat{M}\left(x_{0}=1\right)=\frac{1}{4 !} c_{4} .
$$

Now, from (4.15)

$$
\left|\hat{M}\left(p_{0}\right)-2 \hat{M}\left(x_{0}=1\right) \cos p_{0}\right| \leqq\left|\hat{M}\left(x_{0}=0\right)\right|+4 k_{M} \beta^{7 \varepsilon} .
$$

Since $\operatorname{Im} p_{0} \geqq-5 \log \beta,\left|\cos p_{0}\right| \geqq \sinh (-5 \log \beta)$ and hence,

$$
\begin{aligned}
\mid \hat{M}\left(p_{0}\right)+\tilde{L}_{\chi \chi_{1}} & \tilde{H}_{2}\left(1+\tilde{T}_{2}\right)^{-1} \tilde{L}_{\chi_{1} \chi} \mid \\
& \geqq 2\left|\hat{M}\left(x_{0}=1\right)\right| \sinh (-5 \log \beta)-\left(\left|\hat{M}\left(x_{0}=0\right)\right|+4 k_{M} \beta^{7 \varepsilon}+a \beta^{7 \varepsilon}\right)
\end{aligned}
$$

and we can choose $\beta<\beta_{11} \leqq \beta_{10}$ such that the right hand side is bigger than $c_{4} /(5 ! \beta)$.

The proof of Theorems 2.17 and 2.18 are very similar to the ones for Theorems 2.9 and 2.11, after using the bound in Theroem 2.16. We therefore omit them.

Proof of Theorem 2.20. From (2.15), $\tilde{G}_{\chi_{1} \chi_{1}}=\tilde{F}_{\chi_{1} \chi_{1}}-\tilde{G}_{\chi_{1} \chi} \tilde{\Gamma}_{\chi \chi} \tilde{G}_{\chi_{\chi_{1}}}$ and the possible singularities of $\tilde{G}_{\chi_{1} \chi_{1}}$ on $0 \leqq \operatorname{Im} p_{0} \leqq-7(1-\varepsilon) \log \beta,\left|\operatorname{Re} p_{0}\right| \leqq \pi$ are $p_{0}=i m_{0}, i m_{1}$ and

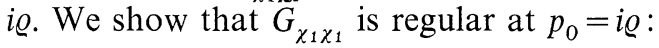

$$
\begin{aligned}
\lim _{p_{0} \rightarrow i \varrho}\left(p_{0}-i \varrho\right) \tilde{G}_{\chi_{1} \chi_{1}}= & \lim _{p_{0} \rightarrow i \varrho}\left(p_{0}-i \varrho\right) \tilde{F}_{\chi_{1} \chi_{1}} \\
& -\tilde{G}_{\chi_{1} \chi}(i \varrho) \tilde{G}_{\chi \chi_{1}}(i \varrho) \lim _{p_{0} \rightarrow i \varrho}\left(p_{0}-i \varrho\right) \tilde{\Gamma}_{\chi \chi} .
\end{aligned}
$$

On the other hand, from (2.22)

$$
\lim _{p_{0} \rightarrow i \varrho}\left(p_{0}-i \varrho\right) \tilde{\Gamma}_{\chi \chi}=\tilde{L}_{\chi \chi_{1}}(i \varrho) \tilde{L}_{\chi 1 \chi}(i \varrho) \lim _{p_{0} \rightarrow i \varrho}\left(p_{0}-i \varrho\right) \tilde{F}_{\chi_{1} \chi_{1}} .
$$

Since

$$
\begin{aligned}
\tilde{L}_{\chi \chi_{1}} & =\tilde{\Gamma}_{\chi \chi} \tilde{G}_{\chi \chi_{1}} \tilde{\Phi}_{\chi_{1} \chi_{1}}=\left(\tilde{L}_{\chi \chi_{1}} \tilde{F}_{\chi_{1 \chi_{1}}} \tilde{L}_{\chi_{1 \chi}}+\tilde{M}\right) \tilde{G}_{\chi \chi_{1}} \tilde{\Phi}_{\chi_{1} \chi_{1}} \\
& =-\tilde{L}_{\chi \chi_{1}} \tilde{L}_{\chi_{1} \chi} \tilde{G}_{\chi \chi_{1}}+\tilde{M} \tilde{G}_{\chi_{1}} \tilde{\Phi}_{\chi_{1} \chi_{1}},
\end{aligned}
$$

we have (remembering that

$$
\left.\tilde{\Phi}_{\chi_{1} \chi_{1}}(i \varrho)=0\right): \tilde{L}_{\chi_{1} \chi}(i \varrho)=-1 / \tilde{G}_{\chi_{1}}(i \varrho) .
$$


Taking (4.17) and (4.18) into (4.16) shows that $\lim _{p_{0} \rightarrow i \varrho}\left(p_{0}-i \varrho\right) \tilde{G}_{\chi_{1} \chi_{1}}=0$. Thus, the only possible singularities are $p_{0}=i m_{0}, i m_{1}$. We show that these are indeed singularities of $\tilde{G}_{\chi_{1} \chi_{1}}$. For suppose $\tilde{G}_{\chi_{1} \chi}$ is regular at $p_{0}=i m_{1}$. Then, from $\tilde{L}_{\chi_{1} \chi}=\tilde{\Phi}_{\chi_{1} \chi_{1}} \tilde{G}_{\chi_{1} \chi} \tilde{\Gamma}_{\chi \chi}$ we would get $\tilde{L}_{\chi_{1} \chi}\left(\operatorname{im}_{1}\right)=0$, which is impossible due to Theorem 2.15 since $\left|m_{1}-m_{2}\right|=O(\beta)$. Similarly, if $\tilde{G}_{\chi_{1} \chi}$ is regular at $p_{0}=i m_{0}$ then $\tilde{L}_{\chi_{1} \chi}\left(i m_{0}\right)=0$. But recall that $\left|\tilde{L}_{\chi_{1} \chi}\left(x_{0}\right)\right| \leqq 64 C_{\chi_{1} \chi}\left(\frac{\beta}{\beta_{6}}\right)^{7\left|x_{0}\right|}$ (from the proof of Theorem 2.13) and $\lim _{\beta \rightarrow 0}(1 / \beta) \hat{L}_{\chi_{1} x}\left(x_{0}=0\right)=4 / d$ (proof of Theorem 2.15). Thus, from

$$
\left|\tilde{L}_{\chi_{1} \chi}\left(i m_{0}\right)-\tilde{L}_{\chi_{1} \chi}\left(x_{0}=0\right)\right| \leqq 64 C_{\chi_{1} \chi} \sum_{x_{0} \neq 0}\left(\frac{\beta}{\beta_{6}}\right)^{7\left|x_{0}\right|} e^{m_{0}\left|x_{0}\right|}
$$

and the fact that $m_{0} \sim-4 \log \beta$ as $\beta \rightarrow 0$, it is clear that $\tilde{L}_{\chi_{1} x}\left(i m_{0}\right) \neq 0$ if $\beta>0$ is small enough.

Proof of Theorem 2.21. Introduce the function

$$
\tilde{F}_{\varphi \varphi}^{(1)}=\tilde{G}_{\varphi \varphi}+\tilde{G}_{\varphi \chi} \tilde{\Gamma}_{\chi \chi} \tilde{G}_{\chi \varphi}+\tilde{F}_{\varphi \chi_{1}} \tilde{\Phi}_{\chi_{1 \chi}{ }_{1}} \tilde{F}_{\chi_{1} \varphi},
$$

where

$$
\tilde{F}_{\varphi \chi_{1}}=\tilde{G}_{\varphi \chi_{1}}+\tilde{G}_{\varphi \chi} \tilde{\Gamma}_{\chi \chi} \tilde{G}_{\chi \chi_{1}} .
$$

By going to the finite volume approximation, one can show by direct but tedious calculations that if $x_{0} \leqq q<y_{0}$,

$$
\left.\frac{\partial^{m}}{\partial w_{q}^{m}} \hat{F}_{\varphi \varphi}^{(1)}\left(x_{0}, y_{0} ; \Lambda\right)\right|_{w_{q}=0}=0 \text { for } 0 \leqq m \leqq 6 .
$$

Thus, $\tilde{F}_{\varphi \varphi}^{(1)}$ is analytic on $\left|\operatorname{Im} p_{0}\right|<-7(1-\varepsilon) \log \beta$ and the only possible singularities

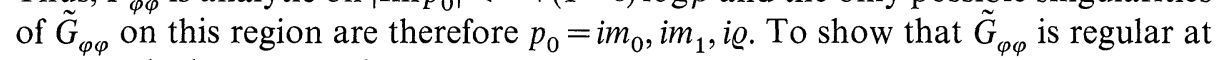
i@, we calculate

$$
\begin{aligned}
0= & \lim _{p_{0} \rightarrow i \varrho}\left(p_{0}-i \varrho\right) \tilde{G}_{\varphi \varphi}+\tilde{G}_{\varphi \chi}(i \varrho) \tilde{G}_{\chi \varphi}(i \varrho) \lim _{p_{0} \rightarrow i \varrho} \tilde{\Gamma}_{\chi \chi} \\
& +\left[\lim _{p_{0} \rightarrow i \varrho}\left(p_{0}-i \varrho\right) \tilde{F}_{\varphi \chi_{1}}\right]\left[\lim _{p \rightarrow i \varrho} \frac{\tilde{\Phi}_{\chi_{1} \chi_{1}}}{\left(p_{0}-i \varrho\right)}\right]\left[\lim _{p_{0} \rightarrow i \varrho}\left(p_{0}=i \varrho\right) \tilde{F}_{\chi_{1} \varphi}\right]
\end{aligned}
$$

Notice that e.g.

$$
\lim _{p_{0} \rightarrow i \varrho}\left(p_{0}-i \varrho\right) \tilde{F}_{\varphi \chi_{1}}=\tilde{G}_{\varphi \chi}(i \varrho) \tilde{G}_{\chi \chi_{1}}(i \varrho) \lim _{p_{0} \rightarrow i \varrho}\left(p_{0}-i \varrho\right) \tilde{\Gamma}_{\chi \chi},
$$

hence

$$
\lim _{p_{0} \rightarrow i \varrho}\left(p_{0}-i \varrho\right)^{-1} \tilde{\Phi}_{\chi_{1} \chi_{1}}=-\left[\tilde{G}_{\chi_{1} \chi}(i \varrho) \tilde{G}_{\chi \chi_{1}}(i \varrho) \lim _{p_{0} \rightarrow i \varrho}\left(p_{0}-i \varrho\right) \tilde{\Gamma}_{\chi \chi}\right]^{-1}
$$

Taking (4.20), (4.21) into (4.19) completes the proof of Theorem 2.21.

Acknowledgements. I would like to thank Erhard Seiler for some discussions and reading the manuscript, and Prof. W. Zimmermann for the hospitality at the Max-Planck-Institut, where this work was carried out. 


\section{References}

1. Fritzsch, H., Gell-Mann, M.: Current algebra : quarks and what else? In: Proc. XVI Intern. Conf. on High Energy Physics, Vol. 2, pp. 135-165. Chicago: Nat. Acc. Lab. 1972

2. Kogut, J., Sinclair, D.K., Susskind, L.: A quantitative approach to low-energy quantum chromodynamics. Nucl. Phys. B114, 199-236 (1976)

3. Berg, B., Billoire, A.: Glueball spectroscopy in $4-d \mathrm{SU}(3)$ lattice gauge theory. Preprint DESY 82-079 (Dec. 1982)

4. Münster, G.: Physical strong coupling expansion parameters and glueball mass ratios. Preprint BUTP 21/1982 (Sept. 1982)

5. Schor, R.S.: Existence of glueballs in strongly coupled lattice-gauge theories. Nucl. Phys. B222, $71-82(1983)$

6. Schor, R.S.: The energy-momentum spectrum of strongly coupled lattice gauge theories. Preprint MPI 15/83 (March 1983) (to appear in Nucl. Phys.)

7. Koch, H.: Irreducible kernels and bound states in $\lambda \mathscr{P}(\varphi)_{2}$ models. Ann. Inst. Henri Poincaré A31, 173-234 (1979)

8. Osterwalder, K., Seiler, E.: Gauge field theories on a lattice. Ann. Phys. 110, 440-471 (1978)

9. Schor, R.S.: The particle structure of $v$-dimensional Ising models at low temperatures. Commun. Math. Phys. 59, 213-233 (1978)

Communicated by K. Osterwalder

Received August 17, 1983 
